\title{
Gut Reactions: Breaking Down Xenobiotic-Microbiome Interactions
}

\author{
Gerard Clarke, Kiran V. Sandhu, Brendan T. Griffin, Timothy G. Dinan, John F. Cryan, and Niall P. Hyland \\ APC Microbiome Ireland (G.C., K.V.S., B.T.G., T.G.D., J.F.C., N.P.H.), INFANT Research Centre (G.C.), Department of Psychiatry and \\ Neurobehavioural Science (G.C., T.G.D.), School of Pharmacy (B.T.G.), and Departments of Anatomy and Neuroscience (J.F.C.), \\ Pharmacology and Therapeutics (N.P.H.), and Physiology (N.P.H.), University College Cork, Cork, Ireland
}

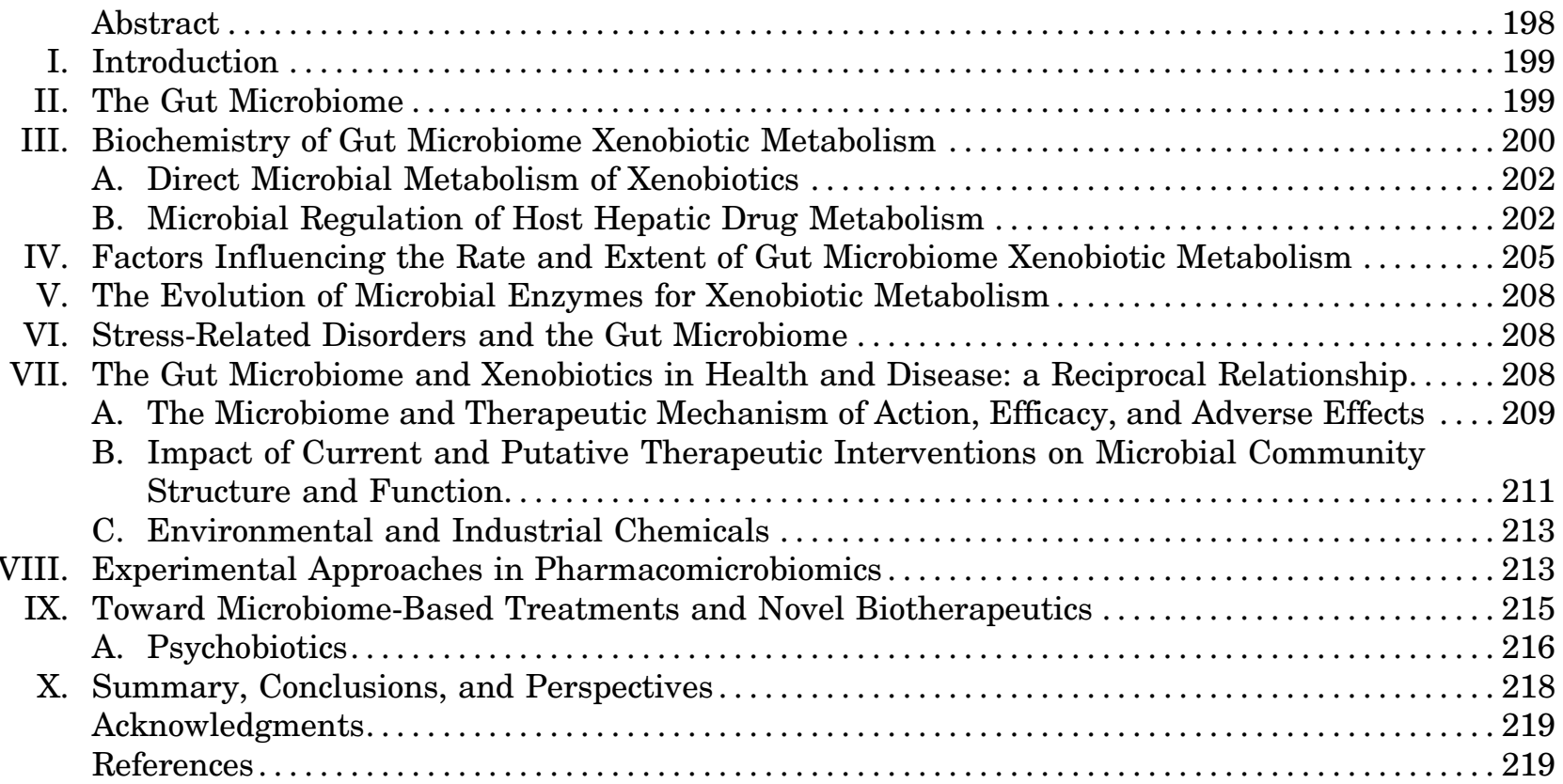

\begin{abstract}
The microbiome plays a key role in health and disease, and there has been considerable interest in therapeutic targeting of the microbiome as well as mining this rich resource in drug discovery efforts. However, a growing body of evidence suggests that the gut microbiota can itself influence the actions of a range of xenobiotics, in both beneficial and potentially harmful ways. Traditionally, clinical studies evaluating the pharmacokinetics of new drugs have mostly ignored the important direct and indirect effects of the gut microbiome on drug metabolism and efficacy. Despite some important observations from xenobiotic metabolism in general, there is only an incomplete understanding of the scope of influence of the microbiome specifically on drug metabolism and absorption, and how this might influence systemic concentrations of parent
\end{abstract}

compounds and toxic metabolites. The significance of both microbial metabolism of xenobiotics and the impact of the gut microbiome on host hepatic enzyme systems is nonetheless gaining traction and presents a further challenge in drug discovery efforts, with implications for improving treatment outcomes or counteracting adverse drug reactions. Microbial factors must now be considered when determining drug pharmacokinetics and the impact that an evolving and dynamic microbiome could have in this regard. In this review, we aim to integrate the contribution of the gut microbiome in health and disease to xenobiotic metabolism focusing on therapeutic interventions, pharmacological drug action, and chemical biotransformations that collectively will have implications for the future practice of precision medicine.

Address correspondence to: Dr. Gerard Clarke, Department of Psychiatry and Neurobehavioural Science and APC Microbiome Ireland, 1.15 Biosciences Building, University College Cork, Cork, Ireland. E-mail: g.clarke@ucc.ie APC Microbiome Ireland is supported by Science Foundation Ireland [Grant SFI/12/RC/2273] https://doi.org/10.1124/pr.118.015768. 


\section{Introduction}

The principles of xenobiotic metabolism, which is defined as the metabolism of ingested exogenous molecules, emphasize the role of the liver as the predominant site of biotransformation after ingestion by the host. Within this convention, the liver is anatomically, morphologically, and physiologically specialized as a metabolic organ and unrivalled in metabolic capacity in comparison with extrahepatic host sites of chemical transformation (Michalopoulos, 2007). This viewpoint overlooks the fact that before orally delivered compounds reach the liver, an increasing number are first exposed to the gut microbiota and their associated collection of metabolic enzymes (which exceeds the repertoire of the liver) (Li et al., 2014). Indirectly, metabolites produced by the gut microbiome can also tune the expression and function of key liver enzymes such as those in the pivotal cytochrome P450 (CYP) superfamily. Thus, the metabolism of many clinically used drugs is likely to be influenced by either direct or indirect effects of the gut microbiome.

In this review, we first outline the current knowledge of the gut microbiome in health and disease before focusing on the metabolic activities associated with this community of microbes residing in the gastrointestinal tract and their aggregate genomes. The collective direct and indirect metabolic influence of these microbes in the gastrointestinal tract is evaluated with regard to the chemical modification of pharmaceutical compounds, dietary components, and environmental agents, and the implications for host health. This is considered within the context of our expanding knowledge of the role played by the gut microbiota in health and disease, host-microbial interactions, and the reciprocal relationship between xenobiotics and the gut bacteria involved in their metabolism. We also discuss the potential therapeutic implications arising from these observations before providing recommendations to guide a currently neglected but growing area of research toward therapeutic dividends and the improvement of human health.

\section{The Gut Microbiome}

The gastrointestinal tract is inhabited by a vast array of microbes-bacteria, fungi, yeasts, archaea, and viruses-termed the gut microbiota (Grenham et al., 2011). The bacterial division of this consortium is best studied and reaches the highest density in the large intestine, with recent estimates of $10^{13}$ bacterial cells in the human colon (Sender et al., 2016a,b). This abundance reflects the optimal bacterial growth conditions in the large intestine, with a mean $\mathrm{pH}$ of 6.4 to 7 , and the high density of bacteria residing there in turn confers this region with a lower redox potential (Sousa et al., 2008). The large intestine is thus an important host site for microbial metabolism, a convenient truth that fortunately aligns with our reliance on the analysis of stool samples to gain insights into our microbial inhabitants (Fraher et al., 2012; Claesson et al., 2017). However, these samples are not representative of the microbiome of more proximal regions of the large intestine, and the nonuniform localization and spatial organization of the gut microbiota along the gastrointestinal tract require further evaluation in terms of the functional implications for microbial xenobiotic metabolism (Tropini et al., 2017). This overreliance on fecal samples, due in part to ease of collection and availability, means we still have an incomplete understanding of the impact of microbial activity in different regions of the gastrointestinal tract and the implications of regionspecific microbial metabolism for health and disease. For example, metabolism of dietary methylamines also occurs in the small intestine with differential profiles of caecal and fecal metabolism (Hoyles et al., 2018b). Moreover, microbes in the small intestine may be an important site for lipid metabolism (Martinez-Guryn et al., 2018) and are enriched in functions specific for the metabolism of simple carbohydrates (Zoetendal et al., 2012; Stremmel et al., 2017). Neither is the gut microbiota homogenous moving from the lumen to the mucosa, instead existing as a type of gradient with colonic mucosa-associated and luminal microbial communities potentially giving rise to niche metabolic capabilities even at seemingly geographically identical gastrointestinal sites (Galley et al., 2014; Donaldson et al., 2016). One recent example of this pertains to the mucin utilizer, Peptostreptococcus russellii, which produces indoleacrylic acid from tryptophan, important for the promotion of enhanced intestinal epithelial barrier function and the suppression of host inflammation (Wlodarska et al., 2017). Microbial enzymes residing in the gut lumen can in turn, for example, deconjugate conjugated cathecholamines with ensuing physiologic implications for colonic function such as water transport (Asano et al., 2012).

Focusing on actual numbers and relative abundances of bacterial cells frequently obscures the importance of their collective genome in the gut ecosystem-the gut microbiome. With an aggregate gene catalog exceeding the human genome by more than a factor of 100 (Qin et al., 2010), the gut microbiome exhibits a diverse functional repertoire, and the encoded metabolic activities of these microbial genes equate to a large metabolic capacity far in excess of its counterpart host genome (Lynch and Pedersen, 2016). According to both 16S rRNA gene sequencing and metagenomic shotgun

ABBREVIATIONS: 5-ASA, 5-aminosalicylate; 5-FU, 5-fluorouracil; CYP, cytochrome P450; IBD, inflammatory bowel disease; miRNA, microRNA; NSAID, nonsteroidal anti-inflammatory drug; PPI, proton pump inhibitor; PUFA, polyunsaturated fatty acid; TMA, trimethylamine; TMAO, TMA-N-oxide. 
sequencing of stool samples, the composition of the adult gut microbiota is dominated by the phyla Firmicutes, Actinobacteria, and Bacteroidetes with lower relative abundances of Verrucomicrobia and Proteobacteria (Zhernakova et al., 2016). Although the interindividual variation in microbiota composition is considerable such that an accurate definition of the precise composition of a healthy human microbiota remains elusive, recent evidence supports a shared global core microbiota consisting of 14 different genera with medication use making an important contribution to microbiota compositional variation (Falony et al., 2016). It should be noted that there remains to be a consensus on actual membership of the core taxa present in the gut microbiome, and different sequencing and analysis approaches will give different results. The study by Falony et al. (2016) also looked at different populations than earlier studies by Turnbaugh et al. (2009) and Qin et al. (2010).

In contrast to this interindividual variation, a typical healthy adult gut microbiota is characterized by both high compositional diversity and stability (Clarke et al., 2014b). Unlike the host genome, the gut microbiome is readily modifiable (e.g., by diet or antibiotic usage), and this can have important implications for expansion or contraction of its metabolic functions. Prebiotics such as short chain fructooligosaccharides can promote the production of equol, an isoflavandiol nonsteroidal estrogen of importance in cardiovascular, bone, and menopausal health, and whose production varies depending on microbiota composition (Setchell et al., 2002). A more extreme example of the modifiable nature of the gut microbiome lies in the transfer of bacterial genes encoding porphyranases, agarases, and associated proteins from marine red algae to bacteria residing in the gastrointestinal tract of Japanese individuals as a consequence of their seaweed consumption (Hehemann et al., 2010). Unfortunately, the flip side of this coin-loss of function-is most likely the more common scenario, and the low-fiber Western diet is held partially responsible for the lower diversity and the possible extinction of important taxa from the gut microbiota of Western populations (Sonnenburg et al., 2016). Reduced microbial gene richness is common in a range of metabolic and hepatic diseases, with inflammation (Cotillard et al., 2013; Le Chatelier et al., 2013; Qin et al., 2014; Hoyles et al., 2018a) and oxygen availability/gradients playing an important role (Albenberg et al., 2014; Schmidt and Kao, 2014; Friedman et al., 2018). The collateral damage inflicted by excessive or inappropriate antibiotic usage on gut microbiota composition is another example currently being debated (Blaser, 2016).

Despite these concerns over under-represented microbes and/or reduced microbial gene richness, the importance of the dictum that structure begets function for the role of gut microbiota composition in xenobiotic metabolism is difficult to gauge given that members of this bacterial ecosystem may exhibit both functional redundancy and pleiotropy (Clarke et al., 2014b; Moya and Ferrer, 2016). As indicated above, colonization of bacteria increases to highest levels in the large intestine such that sites with a higher exposure to xenobiotics in fact may have a lower potential for microbial metabolism, such as the small intestine. However, this needs to be considered in context of the less well studied smallintestinal microbiota, due in part to the difficulty in obtaining relevant samples.

Consideration of the gut microbiome and xenobiotic metabolism also needs to take account of the variation of the gut microbiome at the extremes of life in comparison with the reported stability during adulthood in healthy individuals. Data indicate a narrowing of diversity and distinctive microbiota configurations associated with ageing in long-stay care individuals compared with that of community dwellers (Claesson et al., 2012; Jeffery et al., 2016). This is a time of life with high parallel medication and dietary supplement consumption, although adverse health events are rarely considered with regard to this narrowing microbiota diversity (O'Dwyer et al., 2016; Walsh et al., 2016; Locquet et al., 2017). Conversely, but equally important, the infant gut is largely thought to be sterile in utero and colonized during birth and postnatally with a trajectory toward an adult-like complexity taking approximately 3 years (Clarke et al., 2014a; Perez-Munoz et al., 2017). There is also a divergence in intestinal microbiome assembly between different birth modes (Bokulich et al., 2016), between breast- and formula-fed infants (Clarke et al., 2014a), and between preterm and full-term infants, with implications for the associated microbial metabolite profile (Hill et al., 2017). Other important factors include the antibiotic usage in early life (Bokulich et al., 2016; Korpela et al., 2018) and malnutrition (Million et al., 2017). Of course, during adulthood there are also a number of factors that shape or distort the gut microbiome in the colonic metabolic niche, reviewed in detail elsewhere, such as diet (Portune et al., 2017; Sandhu et al., 2017; Shanahan et al., 2017), exercise (Campbell and Wisniewski, 2017; Barton et al., 2018), geographical location (Dikongue and Segurel, 2017; Quigley, 2017), host genetics (Kurilshikov et al., 2017), and the experience of stress (Moloney et al., 2014; Martin and Mayer, 2017). The ability of the gut microbiome to remodel and persist in the face of insults such as antibiotic use is probably only matched in the host by the regenerative capacity of the liver (Michalopoulos, 2007) (Fig. 1, A and B).

\section{Biochemistry of Gut Microbiome Xenobiotic Metabolism}

Although the collective metabolic activity of the gut microbiome is just beginning to be appreciated with the emergence and growth of the pharmacomicrobiomic concept (Saad et al., 2012; ElRakaiby et al., 2014), the 

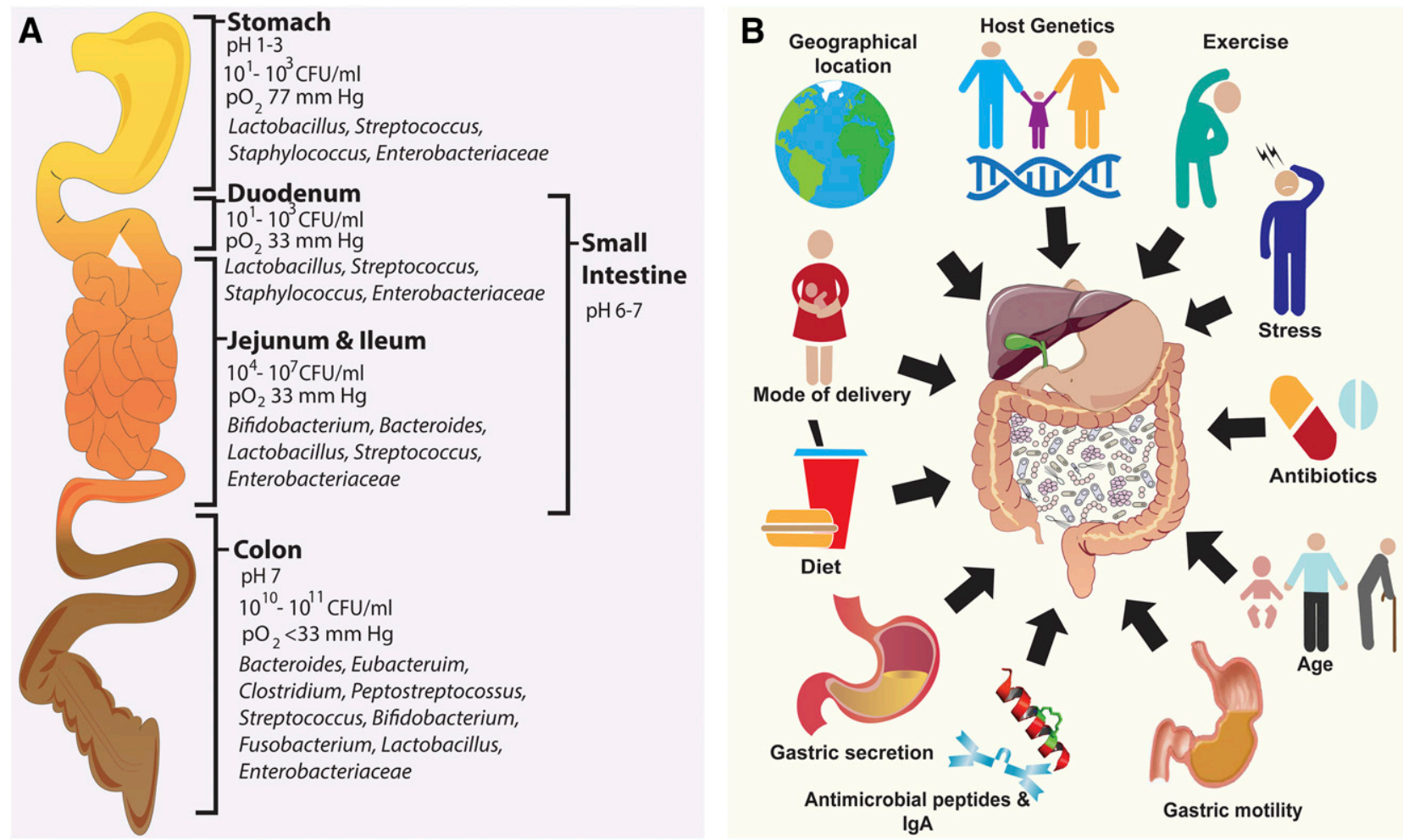

Fig. 1. (A) Metabolic niches in the gut microbiome. The localization and spatial organization of the gut microbiota are not uniform along the gastrointestinal tract. This dynamic gut ecosystem consists of many unique features, such as microniches, $\mathrm{pH}$ gradients, and dynamic microbe-tissue interactions of relevance for microbial biotransformations. The highest density of bacteria is present in the large intestine, with recent estimates of $10^{13}$ bacterial cells in the large intestine associated with microbial genes encoding a broad range of enzymes necessary for xenobiotic biotransformation. These bacteria are likely most important for pharmacomicrobiomics and reside in a reaction chamber with a mean $\mathrm{pH}$ of $6.4-7$ and a lower redox potential than other gastrointestinal niches. Oxygen partial pressures along the gastrointestinal tract also contribute to these metabolic niches. (B) Factors affecting the composition and function of the large intestine metabolic niche. The compositional characteristics of the gut microbiome are influenced by a number of factors, with the initial seeding and trajectory toward an healthy adult-like diversity and stability determined by mode of delivery (C-section vs. par vaginum) and early feeding patterns (breast feeding vs. formula feeding). Host genetics also plays a role as does geographical location, whereas stress across the life span may be viewed as a threat to the diversity of the gut microbiome. A Westernized diet is also thought to compromise the integrity of the gut microbiome, whereas increased fiber intake is associated with increased diversity. Exercise might also promote the stability of a health microbiome, although the ageing process is associated with a narrowing diversity, as are many disease states and excessive/ inappropriate antibiotic use. A number of intrinsic factors, reviewed in details elsewhere by Simren et al. (2013) and partially depicted here, also determine the composition of the gut microbiome, including gastric acid secretion, anticommensal sIgA and antimicrobial peptide production, and gastrointestinal motility.

role of individual microorganisms in drug metabolism has long been studied (de la HUERGA and Popper, 1951; Smith and Rosazza, 1974). This was largely in the context of using microbial models to understand mammalian drug metabolism and with the ambition of using microorganisms for the scalable production of drug metabolites (Murphy, 2015). In contrast to mammalian CYPs, which are usually membrane-bound and hemecontaining enzymes, many bacterial CYPs are soluble (Jezequel, 1998). Genome sequencing of Streptomyces and Bacillus spp. highlighted multiple CYP genes with subsequent culture-based investigations indicating, for example, the production of hydroxylated and amidated metabolites from the nonsteroidal anti-inflammatory drug (NSAID) flurbiprofen that both overlapped with, and were distinct from, the mammalian metabolites (Bright et al., 2011). Enzyme induction was also a noted feature in individual bacterial strains with a CYPdependent fatty acid monooxygenase from Bacillus megaterium strongly inducible by phenobarbital (Narhi and Fulco, 1986). Biotransformation of warfarin by Streptomyces rimosus has also been reported (Cannell et al., 1997), whereas the reduction of warfarin to its corresponding alcohol by Nocardia coralline is a stereoselective reaction (Davis and Rizzo, 1982). Of course not all of these bacteria are found in the human gastrointestinal tract, and it has been noted that even in cases where they are, we cannot assume equivalent metabolizing activities achieved under specific laboratory culture conditions will be recapitulated in the more dynamic ecological conditions of the gastrointestinal tract (Jezequel, 1998). They do, however, offer a window into the metabolic potential that resides in the gut microbiome as well as hinting at the implications for drug metabolism and host response. Traditionally, consideration of these clinical implications in terms of pharmacokinetics and therapeutic response was largely confined to drugs undergoing enterohepatic circulation (Roberts et al., 2002; Spanogiannopoulos et al., 2016). The principle of microbiota-mediated drug-conjugate 
metabolism was also well recognized for many drugs such as oral contraceptives. However, it was rarely considered to have a significant impact clinically except in cases of interactions where broad-spectrum antibiotics resulted in transiently reduced bioavailability of the drugs (Masters and Carr, 2009). In contrast, it is really only as our knowledge of the increasing role that a diverse microbiota can have on health, and the enormous metabolic potential, that interest has turned to the more direct effects of the gut microbiome on metabolizing drugs.

\section{A. Direct Microbial Metabolism of Xenobiotics}

Direct microbial biotransformation of xenobiotics occurs following their ingestion, and the nature of these interactions depends on whether the compounds encountered by the gut microbiome are poorly absorbed or are first conjugated by the liver and subsequently reach the intestine via biliary excretion. Once present in the intestinal lumen, an array of bacterial enzymes is capable of a diverse set of chemical reactions. Glucuronides are abundant in the gut and are subject to processing by microbial $\beta$-glucuronidase enzymes, which act to liberate glucuronic acid sugars from conjugated compounds (Pellock and Redinbo, 2017). A recent analysis of the Human Microbiome Project gastrointestinal database is instructive on the complexity of this metabolic function, revealing a total of 3013 microbiome-encoded $\beta$-glucuronidases, which clustered into structural categories with differing functional capacities for various glucuronide substrates (Pollet et al., 2017). Although the extent of this variation is somewhat bewildering, an earlier study neatly demonstrated the important implications arising from these observations in that the propensity for enzyme inhibition varied according to the bacterial provenance of the orthologous enzymes (Wallace et al., 2015). Endogenous glucuronides such as serotonin glucuronide as well as glucuronidated catecholamines are processed in this way (Asano et al., 2012; Hata et al., 2017). Examples of exogenous glucuronides subject to microbial deconjugation include both therapeutic agents such as the colorectal cancer drug irinotecan and the NSAID diclofenac (Roberts et al., 2013), diet-derived carcinogenic compounds such as 2-amino-3-methyl-3H-imidazo [4,5f]quinoline (Humblot et al., 2007), and likely many representatives from the broad polyphenol family of compounds (Piwowarski et al., 2017; Williamson and Clifford, 2017), including dietary flavonoids (Murota et al., 2018).

In addition to the hydrolysis of these glucuronide conjugates, there are also gut microbial polysaccharide lysases, lipases, reductases (azoreductases and nitroreducases), endoglycosidases, transferases, monoand dioxygenases, sulfatases, and glycyl radical enzymes (Claus et al., 2016; Koppel et al., 2017). As for the $\beta$-glucuronidases, some of these enzyme families, such as the glycyl radical enzymes and S-adenosylmethionine enzymes, have multiple family members and likely different substrate specificities and functions (Lehtio and Goldman, 2004; Haft and Basu, 2011; Murphy et al., 2011). This confers a spectrum of functional metabolic properties on the gut microbiome ranging from proteolysis and deconjugation to amine formation and acetylation (Tralau et al., 2015; Wilson and Nicholson, 2017). Taken together, this functional metabolic repertoire includes the capacity for clinically relevant activation (e.g., sulfasalazine) (Peppercorn and Goldman, 1972b), reactivation (e.g., irinotecan/SN-38) (Wallace et al., 2010), and detoxification (e.g., digoxin) (Haiser et al., 2013) of xenobiotics with broad implications for drug action and toxicity (see Table 1). The administration of probiotics such as Lactobacillus reuteri $\mathrm{K} 8$ and Lactobacillus rhamnosus $\mathrm{K} 9$ in mice has also been shown to alter the fecal activity of microbial enzymes such as sulfatase, arylsulfate sulfotransferase, and $\beta$-glucuronidase, with implications for the pharmacokinetics of acetaminophen in the case of the former (K8) probiotic (Kim et al., 2018).

In many cases, as for dietary compounds such as the artificial sweeteners xylitol and stevioside, the precise enzymes carrying out their metabolism or their origin microbial species are unknown (Koppel et al., 2017). For others, the enzymatic pathways and the associated implications are becoming clearer. A chalcone isomerase has been isolated from Eubacterium ramulus, a human fecal anaerobe capable of degrading various flavonoids (Herles et al., 2004). Experimentally, Western-style high-fat diets have been associated with a diminished microbial capacity to metabolize flavonoids through bacterial chalcone synthase, and microbiome-derived flavonoids can ameliorate the rate of postdieting weight gain (Thaiss et al., 2016a; Chilloux and Dumas, 2017). Daidzin and genistin, glycoside isoflavones, are converted to daidzein and genistein by bacterial $\beta$-glucosidases, with the important estrogen equol being the end product following further biotransformation of daidzein (Setchell et al., 2002). Human gut bacteria Adlercreutzia equolifaciens and various Slackia spp. (all related to Eggerthella lenta) have been shown to produce these metabolites (Maruo et al., 2008; Tsuji et al., 2010; Matthies et al., 2012), and the daidzein-induced gene cluster has been described (Tsuji et al., 2010).

\section{B. Microbial Regulation of Host Hepatic Drug Metabolism}

The range of metabolic activity of the gut microbiome outlined above is supplemented via microbial regulation of host enzyme expression and activity in both the liver and large intestine, effectively regulating aspects of host drug absorption and metabolism (see Table 2). The use of germ-free animals has revealed that important metabolic enzymes are either up- or downregulated 


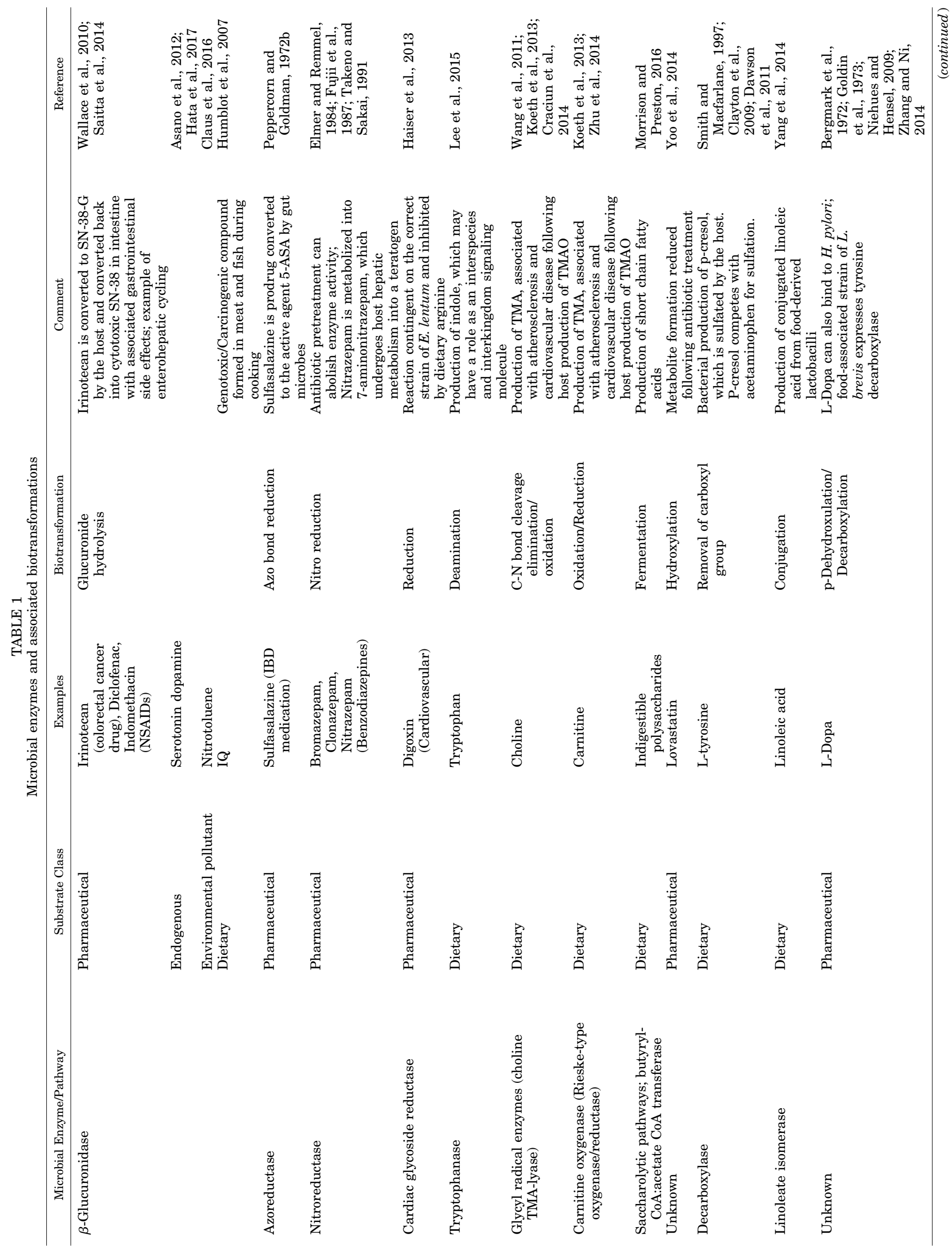

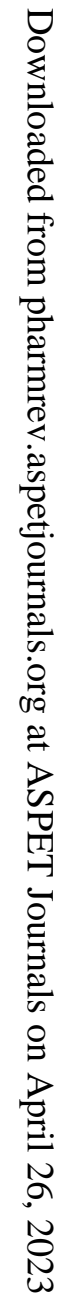




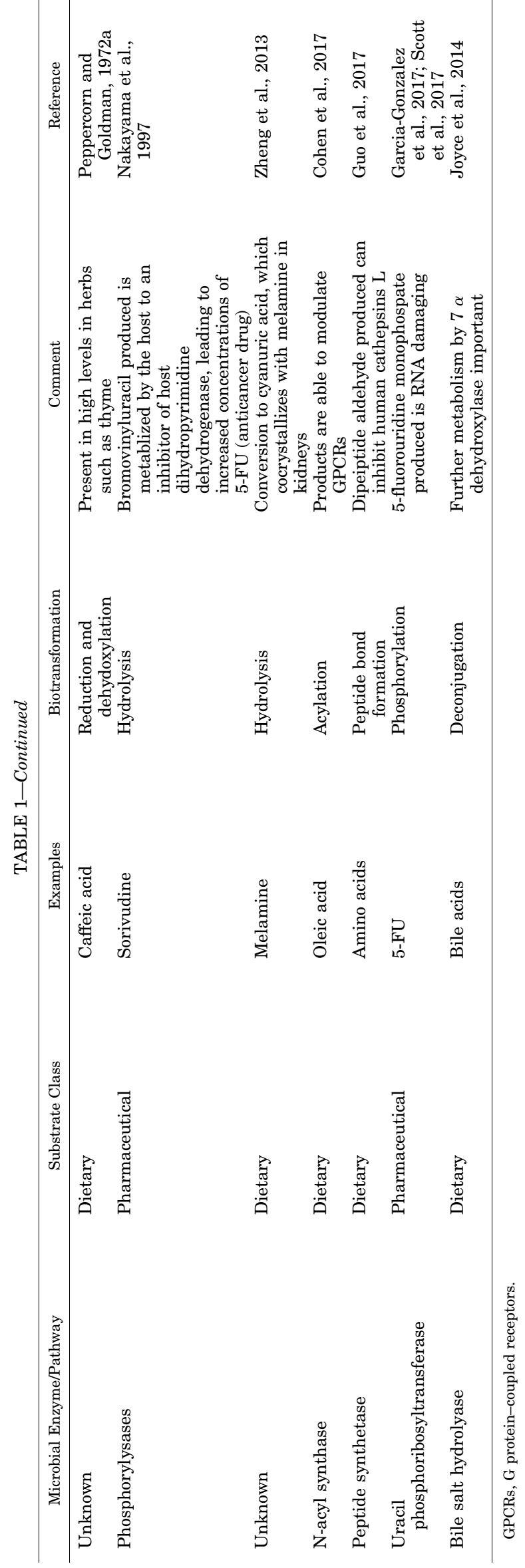

in the absence of a gut microbiome. This includes glutathione transferases (detoxification enzymes), gastrointestinal glutathione peroxidase (reduction of lipid hydroperoxides), epoxide hydrolases (detoxification of genotoxic compounds), $\mathrm{N}$-acetyltransferases (phase II conjugation reactions), and sulfotransferases (detoxification and hormone regulation) (Meinl et al., 2009). Both mRNA and protein expression of CYP3a11 is markedly reduced in the livers of adult germ-free mice (Selwyn et al., 2015b). CYP3a11 is the mouse homolog of human CYP3A4, and this enzyme metabolizes more than $60 \%$ of all drugs. Conversely, the same study showed that gene expression levels of Cyp1a2 (metabolic activation of procarcinogens and deactivation of certain anticancer drugs) and Cyp4a14 (metabolism of fatty acids and eicosanoids) were both substantially increased in germ-free mice. Studies also indicate that the impact of the gut microbiota on hepatic drug-processing genes is enzyme-specific and depends on both age and sex, with expression patterns varying across the life span according to developmental periods, and the most marked effects observed in male mice at 90 days of age (Selwyn et al., 2015a).

Microbial colonization of germ-free animals normalized the expression of Сyp3a and Cyp4a gene clusters to those observed in conventional animals (Selwyn et al., 2016). Administration of VSL3, a probiotic formulation containing eight live strains of bacteria, did not impact on the expression of these genes in germ-free animals but did modulate their expression in conventional animals, suggesting that the composition of the host microbiota can influence the effects of other microbes on expression of host drug-metabolizing enzymes. Moreover, this intervention confirms that less extreme microbiome manipulations, outside of proof-of-principle studies in microbiota-deficient animals, can influence the expression of hepatic drug-processing genes during adulthood. Colonization effects are likely strain specific because monoassociation of germ-free mice with both Lactobacillus plantarum Nizo2877 and Escherichia coli Nissle 1917 decreased the expression of Cyp1a2 mRNA to conventional levels but did not act to restore the decreased expression of Cyp3a11 mRNA. Differential effects for these strains were also noted with increased expression of hepatic Cyp2e1 following monoassociation with $L$. plantarum Nizo2877 but decreased expression following colonization of $E$. coli Nissle 1917 (Jourova et al., 2017).

This transcriptional regulation of such a range of important host drug-processing genes by the gut microbiome may have important implications in terms of microbiome-drug interactions that are not currently routinely considered. This is especially salient in the context of antibiotic usage and the transient depletion of important members of the 
TABLE 2

Microbial regulation of host hepatic cytochrome P450 drug-processing enzymes

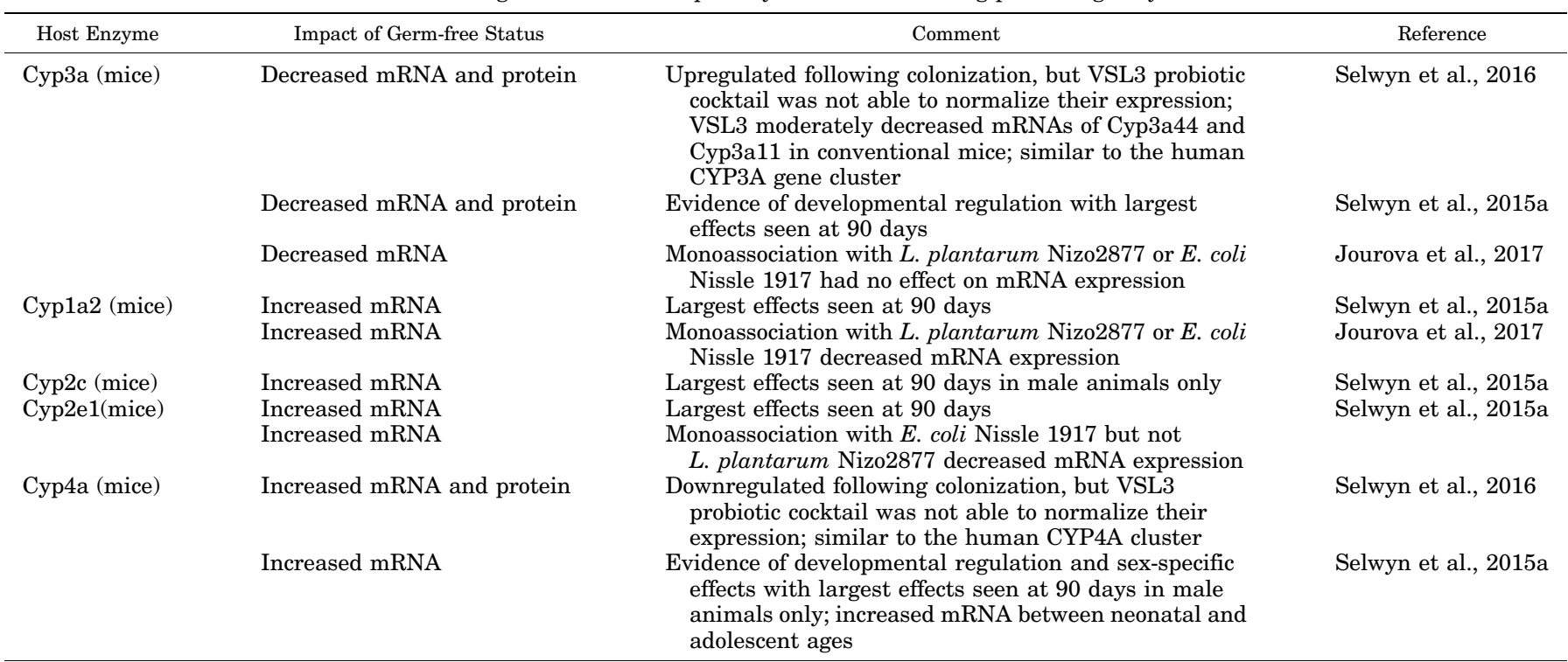

bacterial consortium in the gut microbiota. It has been shown, for example, that ciprofloxacin (a broadspectrum fluoroquinolone antibiotic) reduces hepatic Cyp3a expression and metabolism of the benzodiazepine triazolam (Toda et al., 2009a). This was associated with a reduction in lithocholic acid-producing bacteria, consistent with the suggestion that lithocholic acid influences hepatic CYP expression (Toda et al., 2009b). It should be noted that ciprofloxacin is metabolized in the liver and can directly inhibit CYP1A2, CYP2D6, and CYP3A4 (Bolhuis et al., 2011). Whether poorly absorbed antibiotics that avoid significant hepatic metabolism, such as vancomycin and rifaximin, also indirectly modulate hepatic CYP gene expression via depletion of the gut bacteria is currently unknown. An improved understanding of the consequences of indirect hostmicrobiome drug interactions during these exposures, which are associated with concurrent and relevant drug use, consumption of dietary supplements, or environmental toxins (which might also alter the gut microbiome), is urgently required and should be considered in drug development, safety pharmacology, and pharmacokinetic profiling (Fig. 2).

\section{Factors Influencing the Rate and Extent of Gut Microbiome Xenobiotic Metabolism}

Xenobiotics can become a candidate substrate for direct processing if they are poorly absorbed, appropriately formulated, or indirectly reach the colonic lumen following biliary excretion, which is also a route through which i.v. administered drugs can become subject to chemical transformation by gut microbes. In considering the rate and extent to which drugs will be exposed to direct microbiome metabolism, there are a myriad of additional factors that need to be considered (Hall et al., 1999).
The majority of orally administered drugs are normally absorbed in the upper small intestine, the prime absorptive region of the intestine, assuming that the drug is highly soluble and highly permeable in this region. However, many drugs display poor solubility, which can lead to slow and incomplete absorption with the drug being absorbed from distal regions of the small intestine and/or the colon. Drugs with low permeability through the intestinal membrane, or drugs that are subject to efflux by apically bound efflux proteins (e.g., $\mathrm{P}$ glycoprotein), can prolong drug residence within the intestine, with greater amounts of those drugs reaching distal regions of the intestine (Hall et al., 1999). Finally, it should also be recognized that although microbial abundance is lower in the small intestine, this should not lead to the implicit assumption that it will have a limited impact on the drugs that are predominantly absorbed in this region. On the contrary, in the case in intestinal CYP enzymes, which are present in the intestine, but expressed in vastly lower amounts than the liver, intestinally mediated CYP metabolism can still be the major site of drug metabolism (Hall et al., 1999). For example, in the case of cyclosporine, it is estimated that up to half of the drug is metabolized by intestinal CYP enzymes (Benet and Cummins, 2001), illustrating that relatively low amounts of enzymes in the small intestine could still potentially have a significant impact for orally administered drugs. This needs to be considered in the context that due to our reliance of fecal microbiota assessments, comparatively little is known about the microbiota of the less accessible small intestine (El Aidy et al., 2015).

Oral delivery of advanced drug formulations designed for extended release (e.g., sustained release tablets) is on the increase, circumventing absorption profiles that would usually limit exposure of many drugs to the 

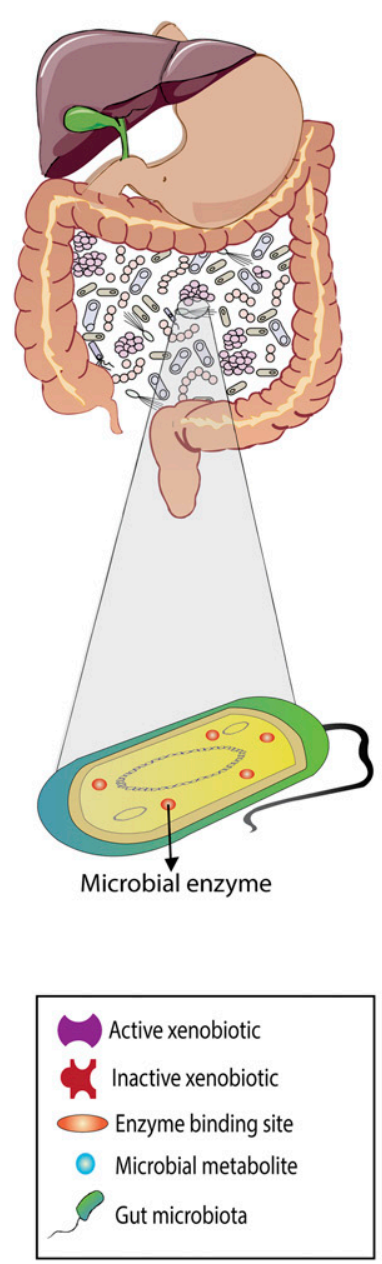

\section{Direct Mechanism}

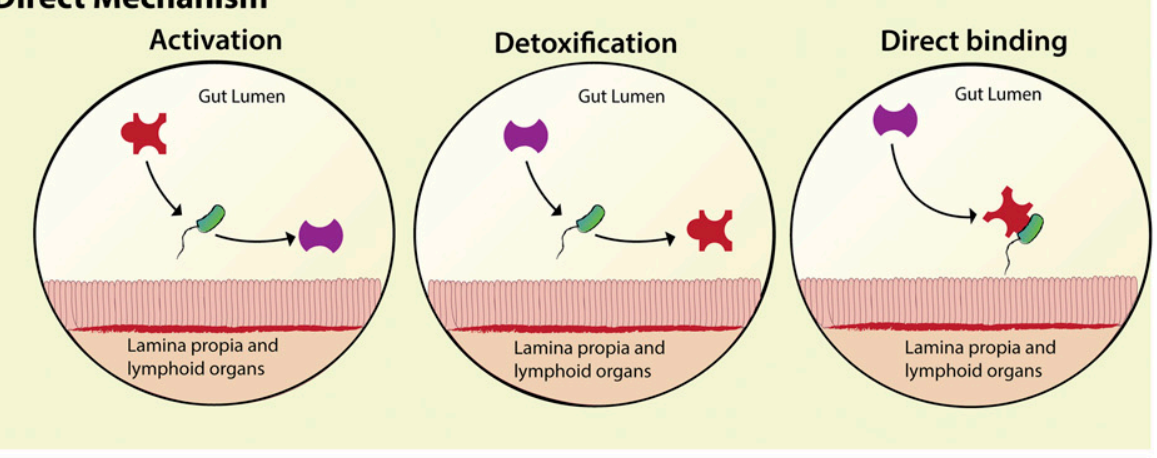

Indirect Mechanism
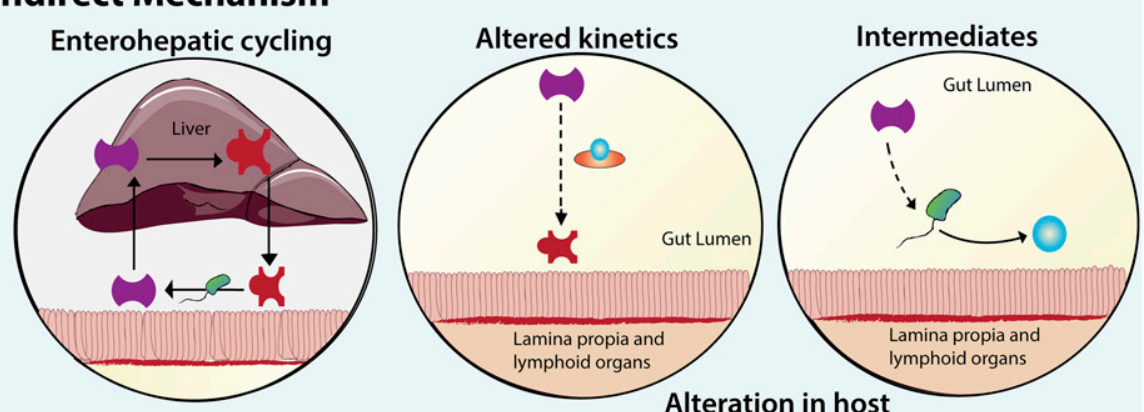

Alteration in host
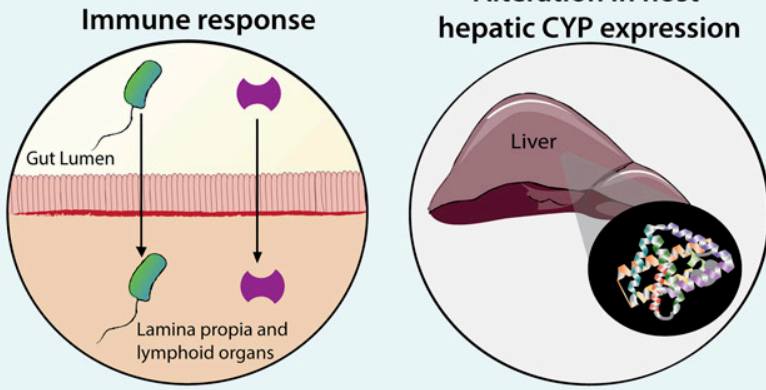

Fig. 2. Metabolic activity of the gut microbiome. There are both direct and indirect mechanisms through which the gut microbiome can influence xenobiotic metabolism to modulate efficacy, absorption, and bioavailability. Direct mechanisms include activation (conversion of prodrugs into active drugs) and deactivation (detoxification). Direct binding of xenobiotics to bacterial cells is also possible (e.g., precursor binding). Indirect mechanisms include enterohepatic cycling, as microbial biotransformation of compounds conjugated in the liver releases the conjugated group, reactivating the compound. The expression of host hepatic CYP genes involved in xenobiotic metabolism is also influenced by the gut microbiome. Microbial metabolites and/or production of pathway intermediates are also important from a pharmacokinetic perspective, as is the impact of bacterial translocation on immune responses in the context of therapeutic efficacy.

colonic gut microbiota (Sousa et al., 2008). This approach has become popular, particularly for the more effective treatment of neuropsychiatric disorders, but, in addition to extending the economic life cycle of drugs (Andrade, 2015), these modified release formulations also increase the scope for drug-microbiome interactions (Enright et al., 2016), and this is a feature rarely appraised for its contribution to bioavailability.

In contrast to the usual objective of host metabolism, biochemical modification of exogenous compounds by our gut microbes is not always geared toward elimination of these foreign compounds. Once a substrate is available for microbial metabolism, there are a number of considerations that may determine its fate and the extent to which it undergoes biotransformation. Much like the host, these can be considered in terms of interindividual and intraindividual variables (Kramer and
Testa, 2008, 2009). Individual-specific genetic makeup of the host genome explains some differences in drug metabolism, and host-genetic influences on gut microbiome composition (Goodrich et al., 2016; Rothschild et al., 2018) are also likely to play a role in influencing the rate and extent of microbial xenobiotic metabolism. Microbial enzymes, such as $\beta$-glucuronidases, can differ in substrate selectivity and activity depending on the bacteria from which they arise (Pollet et al., 2017). Of note, interindividual differences in the abundance of a cytochrome-encoding operon, responsible for the deactivation of the cardiac drug digoxin, have recently been reported, and this operon was specific to drug-metabolizing strains of $E$. lenta and was inducible by the drug it metabolizes (Haiser et al., 2013). Other examples of inducible bacterial enzymes include the lac operon of $E$. coli, which contains genes involved in lactose metabolism (Wilson et al., 2007) that is expressed 
only when lactose is present and glucose is absent. It is currently unclear the extent to which this feature of inducibility, or indeed repression, generalizes to other microbial enzymes important for xenobiotic metabolism or whether constitutive expression is the more common guiding rule in the gastrointestinal tract.

Factors that are known to influence the composition of the gut microbiome, such as age and geography, also overlap with variable drug pharmacokinetics and impact on the relative abundances of genes coding for xenobiotic-metabolizing enzymes (Das et al., 2016; Quigley, 2017). However, our knowledge of interindividual variation of the gut microbiome during health and disease at the taxonomic level is more advanced than our grasp of the functional implications, if any, of these compositional differences. Although studies suggest that we share a stable set of core functions despite these variable gut microbiota profiles (Human Microbiome Project Consortium, 2012), actual metabolic activity of the gut microbiome may diverge from its functional potential (Tanca et al., 2017). Because the activity of the gut microbiota may exhibit resilience despite fluctuating community membership and structure (Song et al., 2015), it is thus not yet clear how many of the variables associated with alterations in the composition and stability of the gut microbiome, including diet (Shanahan et al., 2017), leave their mark on the capacity for xenobiotic metabolism. It is, however, notable that there are examples of disease-associated gut microbiomes, such as depression, that do result in altered behavioral phenotypes and host physiologic characteristics when transferred into animal models (Kelly et al., 2016a). The microbiome-associated metabolite phenylacetic acid has also been shown to influence disease phenotype in nonalcoholic fatty liver disease, contributing to hepatic lipid accumulation (Hoyles et al., 2018a).

Host xenobiotic metabolism is influenced by circadian rhythms (Ozturk et al., 2017), and this may also be true of microbial enzymatic activity because gut microbiota community structure and metabolic activity also feature biologic rhythms, regulated by diet and time of feeding rather than environmental 24-hour light-dark cycles (Voigt et al., 2016; Johnson et al., 2017). Jet lag can disrupt this diurnal microbiota biology, a feature driven by alterations in feeding patterns (Thaiss et al., 2014). Diurnal microbial behavior in turn is thought to influence the programming of the colonic and hepatic circadian transcriptome via fluctuating microbial metabolites with implications, for example, for the hepatic detoxification of acetaminophen that was dependent on the timing of administration (Thaiss et al., 2016b). This was linked to variation in the number of mucosa-associated bacteria in the mouse gut across the circadian period, which was 10 times higher during the dark phase than during the light phase. Circadian rhythm disruption and appetite fluctuations, including carbohydrate craving, are prominent in stress-related psychiatric disorders such as major depression (Otte et al., 2016), which is also associated with gut microbiome alterations (Dinan and Cryan, 2017) (Fig. 3).

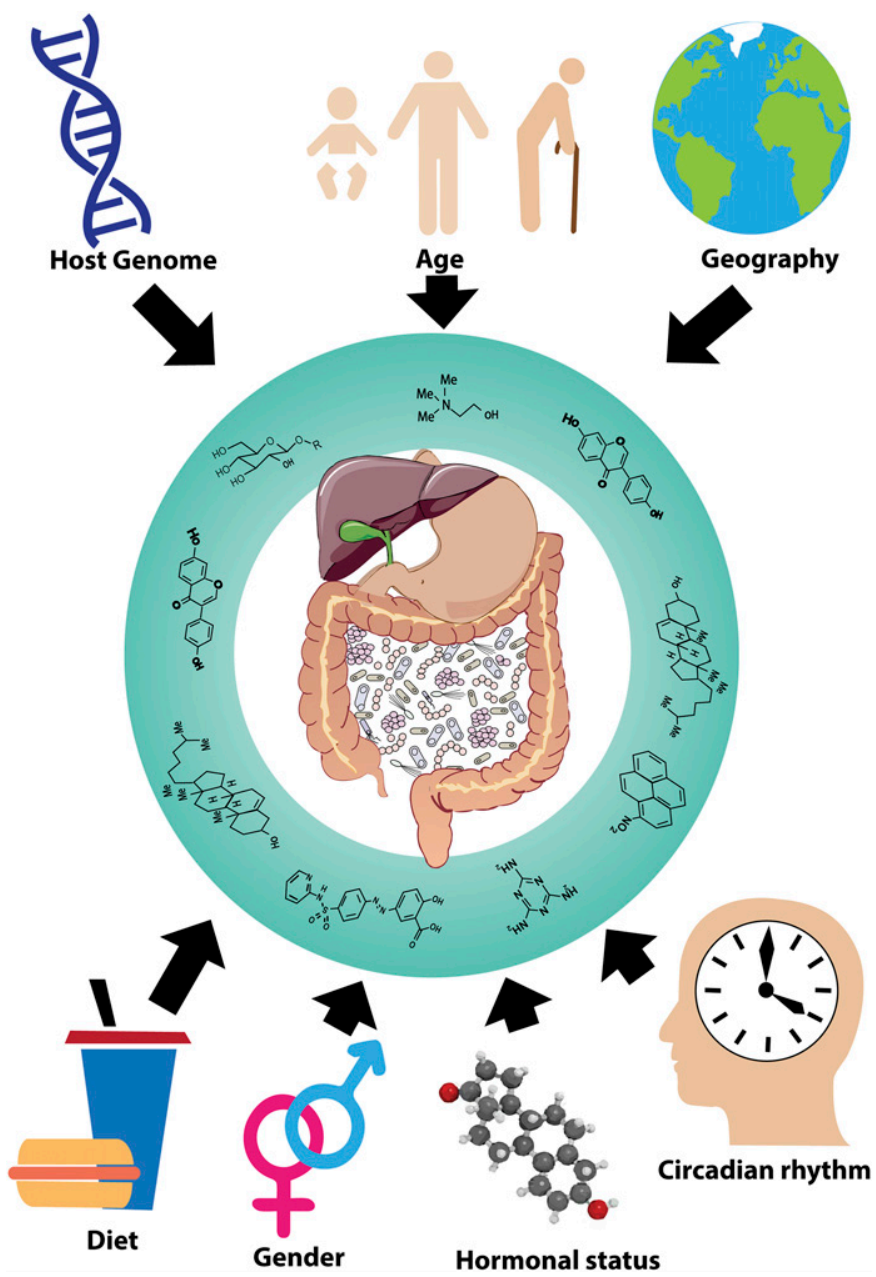

Fig. 3. Factors influencing gut microbiome xenobiotic metabolism. There are a number of considerations that may determine the rate and the extent to which accessible substrates undergo microbial biotransformation. Although compositional differences may not necessarily equate to functional alterations, individual-specific genetic makeup of the host genome explains some differences in gut microbiome characteristics and may affect metabolic outputs. For example, microbial enzymes, such as $\beta$-glucuronidases, can differ in substrate selectivity and activity, depending on the bacteria from which they arise. Other factors known to influence the composition of the gut microbiome, such as age, hormone status, and sex, also overlap with variable drug pharmacokinetics and impact on the relative abundances of genes coding for xenobioticmetabolizing enzymes. Host xenobiotic metabolism is also influenced by circadian rhythms, and this may also be true of microbial enzymatic activity because gut microbiota community structure and metabolic activity also feature biologic rhythms. It is also not yet clear how many of the variables associated with alterations in the composition and stability of the gut microbiome, including diet and geography, leave their mark on the capacity for xenobiotic metabolism. An extreme example of the modifiable nature of the gut microbiome in the context of diet and geography lies in the transfer of bacterial genes encoding porphyranases, agarases, and associated proteins from marine red algae to bacteria residing in the gastrointestinal tract of Japanese individuals as a consequence of their seaweed consumption. 


\section{The Evolution of Microbial Enzymes for Xenobiotic Metabolism}

Host-microbe interactions are frequently viewed from an evolutionary perspective (Davenport et al., 2017). This informs the hologenome theory of evolution with the holobiont encompassing a network of biomolecules derived from both the host plus its associated microbes (Bordenstein and Theis, 2015). Both the host and the microbiome have evolved an extensive metabolic capacity to deal with exogenous molecules with the combined scope impressive in its breadth. Moreover, host-microbe interactions appear critical to both normal and perturbed regulation of metabolic activity, although not all aspects of these actions are mutually beneficial, and the rationale for either the host or the microbiome to have this capability is not always immediately obvious (Patterson and Turnbaugh, 2014). For example, it has been noted that the most common biotransformations carried out by the gut microbiome (hydrolysis and reduction) often serve to undo the oxidative and conjugative chemistry and indeed the intended eliminative purpose of host hepatic drug metabolism (Koppel et al., 2017). As detailed in Table 1 , this can have consequences in terms of deactivating or activating drugs in the case of digoxin (Haiser et al., 2013) and sulfasalazine (Peppercorn and Goldman, 1972b), respectively. Toxic or adverse consequences can also ensue, as is the case for irinotecan (Wallace et al., 2010) or the production of trimethylamine (TMA) from dietary carnitine and choline (Wang et al., 2011; Koeth et al., 2013). In many cases, as in the latter example, host-microbe interactions drive the adverse metabolic consequences with the host responsible for processing of the intermediate TMA to the cardiotoxic TMA-N-oxide (TMAO; see section on VII. A. The Microbiome and Therapeutic Mechanism of Action, Efficacy, and Adverse Effects). In mice, however, TMA is also a chemosignal involved in species-specific social communication, acting to attract mice but repel rats (Li et al., 2013).

An overarching question relates to why either the host or the associated microbiome would evolve enzymes to metabolize substances that they could not expect to come in contact with on a consistent basis. Or, in other words, has microbial biochemistry evolved to counter the vast increase in xenobiotics they encounter both in the body and in environment as consequence of industrialization and the subsequent expansion of the chemical universe? It has been proposed that the answer lies in the functional redundancy of the enzymes, with a broad substrate specificity being a common feature of reactions catalyzed by both host and microbial enzyme (Patterson and Turnbaugh, 2014). The reactions carried out by the gut microbiome frequently result in the release of small molecules or carbon sources that can be used for microbial growth (Koppel et al., 2017). Exposure of the host, in contrast, to a wide array of bacterial metabolites via the hepatic portal circulation demanded the emergence of enzymatic flexibility primarily in the liver. The most expansive enzyme families, such as the $\beta$-glucuronidases, for example, reflect the wide availability of host-derived glucuronide substrates in the gastrointestinal tract. Indeed, these enzymes have an important role in the enterohepatic cycling of endogenous substances such as bile acids (Long et al., 2017). Because much of this chemistry involves the modification of common functional groups, it is perhaps not that surprising that modern pharmaceuticals, dietary components, and environmental pollutants (see Table 1) have become suitable substrates for the expansive range of metabolic functions noted in this review.

\section{Stress-Related Disorders and the Gut Microbiome}

Stress is known to impact on host xenobiotic metabolism, particularly via induction of hepatic drug-metabolizing enzymes possibly mediated by glucocorticoids or noradrenaline (Konstandi, 2013; Pantelidou et al., 2017). Gut microbiome alterations are frequently reported in stressrelated disorders such as depression, anxiety, and irritable bowel syndrome (Rajilic-Stojanovic et al., 2015; Foster et al., 2017). The impact of stress on the already compromised ageing microbiome is currently under consideration and aligns with studies on the impact of stress on the ageing brain at a time of life when chronic stressors are common and in the context of shifting worldwide population demographics (Prenderville et al., 2015; Allen et al., 2017). Understanding the implications of stressmicrobiome interactions, and their functional metabolic consequences and the implications for drug action, is surely now an important research objective (Fig. 4).

\section{The Gut Microbiome and Xenobiotics in Health and Disease: a Reciprocal Relationship}

Although research efforts are ongoing to more precisely define a healthy gut microbiome (Falony et al., 2016; Zhernakova et al., 2016), one of the most frequently reported findings across an array of disorders is a narrowing of gut microbiome diversity often accompanied by more specific but less consistent compositional alterations at various taxonomic levels. This includes central nervous system disorders such as depression (Kelly et al., 2016a) and schizophrenia (Schwarz et al., 2018), metabolic disorders such as obesity (Torres-Fuentes et al., 2017) and diabetes mellitus (Sohail et al., 2017), cardiovascular disorders (Ryan et al., 2015; Winek et al., 2016), inflammatory disorders such as inflammatory bowel disease (IBD) (Sheehan and Shanahan, 2017), rheumatoid arthritis and multiple sclerosis (Forbes et al., 2016), and cancer (Aviles-Jimenez et al., 2017). Many of the therapeutic interventions for such disorders are subject to 


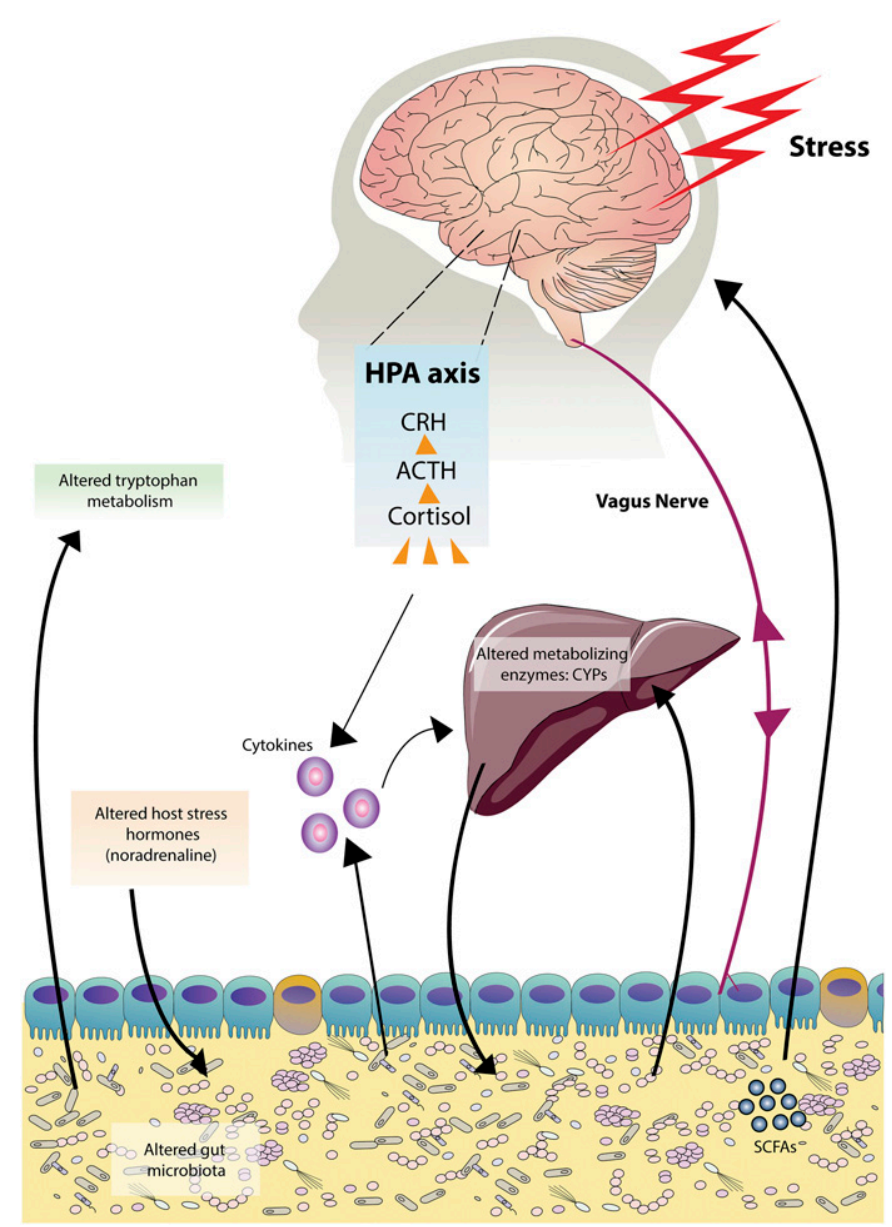

Fig. 4. Stress and the gut microbiome. The gut microbiome recruits the gut-brain axis to signal to the central nervous system. Signaling pathways include the vagus nerve, neuroendocrine and neuroimmune routes, as well as the impact on tryptophan metabolism. Communication along this axis is bidirectional. Although the host response to stress is coordinated by the brain with the central nervous system responding to stressors by structural remodelling of neural architecture (McEwen, 2017), the gut microbiome appears to be equally vulnerable and plastic in its response to homeostatic threats directed at either terminus of the gut-brain axis (Dinan and Cryan, 2017). Hypothalamic-pituitary-adrenal axis programming and responses show a dependency on an intact gut microbiome (Clarke et al., 2014b). Preclinical studies indicate that stress exposure across the life span can impact on gut microbiome composition. This includes early-life prenatal (Golubeva et al., 2015) or postnatal stressors (O'Mahony et al., 2009) as well as stress experienced during adulthood (Bharwani et al., 2016; Burokas et al., 2017; Galley et al., 2017).

biotransformation by our gut microbiome (see Table 3), and the functional implications of these gut microbiome alterations are not currently clear for microbial xenobiotic metabolism. This gap in knowledge surrounding how these disease-associated gut microbiome alterations feed into variations in drug response and toxicity is further compounded by the realization that many of the interventions themselves may also change the composition and function of the gut microbiome.

\section{A. The Microbiome and Therapeutic Mechanism of Action, Efficacy, and Adverse Effects}

Perhaps the best example in the literature that ties the compositional characteristics of the gut microbiome to drug action explicitly via microbial metabolism relates to digoxin, a cardiac glycoside used in the management of cardiovascular disorders. Haiser et al. (2013) elegantly demonstrated that digoxin inactivation was significantly influenced by the presence or absence of metabolizing $E$. lenta strains in the gut microbiota of patients, a feature offered as an explanation for variable digoxin metabolite levels. Digoxin can be used for the treatment of heart failure and atrial fibrillation, common disorders in the elderly who are noted to have reduced elimination of digoxin (Haiser et al., 2013). Despite the important conceptual advance provided by this study and the template offered for the design of future studies in this area, it is also worth noting that digoxin use is on the decline amid the emergence of alternative therapies with preferable safety profiles (Haynes et al., 2008; Eade et al., 2013). Other examples of microbiota-drug interactions of relevance to cardiovascular pharmacology include the hydroxylation of lovastatin, albeit less well-characterized in terms of specific enzyme involvement, in an animal model following coadministration of an antibiotic that resulted in a more extreme depletion of the microbiota that is known to be present in cardiovascular disorders (Yoo et al., 2014).

Choline TMA-lyase, a glycyl radical enzyme, is also widely distributed among gut bacteria and exhibits selectivity for choline cleavage. Choline is an essential nutrient found in a wide variety of foods, but excess conversion to TMA can lead to artherosclerosis as a consequence of further metabolism by host hepatic processing to TMAO (Zeisel and da Costa, 2009; Wang et al., 2011; Koeth et al., 2013; Craciun et al., 2014). TMAO can be produced from dietary TMA via metabolic retroconversion (Hoyles et al., 2018b) and is predicted to be produced from betaine (Jameson et al., 2016). TMA is also produced by carnitine oxygenase, a Rieske-type protein, using carnitine found in red meat as a substrate (Zhu et al., 2014). Choline TMA-lyase may be more vulnerable to compositional variations in the gut microbiome, and a recent study indicated that it was only present in $26 \%$ of individuals, while also noting that further studies were required to characterize the TMA-producing capacity of bacterial communities in at-risk groups (Rath et al., 2017) and consequential effects on cardiovascular health. Interestingly, although red meat has been negatively implicated as a substrate source, commentators have pointed out that fish and other seafood presumed to be beneficial to health also contain significant amounts of TMA precursors as well as free TMAO (Landfald et al., 2017). As TMAO is excreted into urine via the kidney, and circulating levels of TMAO increase significantly in patients suffering from chronic kidney disease, there is a line of thought suggesting plasma TMAO concentrations may be a better marker of impaired renal function associated with atherosclerosis of the renal vasculature rather than a direct cause of 
TABLE 3

Clinical specialties, therapeutic interventions, and pharmacomicrobiomics

\begin{tabular}{|c|c|c|c|}
\hline Specialty & Drug Class & Example & Reference \\
\hline \multirow[t]{5}{*}{ Oncology } & Immune checkpoint inhibitor & Anti-programmed cell death 1 protein & $\begin{array}{l}\text { Gopalakrishnan et al., 2018; } \\
\text { Routy et al., } 2018\end{array}$ \\
\hline & & Anti-CTLA-4 antibodies & Alexander et al., 2017 \\
\hline & Antineoplastic enzyme inhibitor & Irinotecan & Wallace et al., 2010 \\
\hline & Anthracycline & Doxorubicin & Yan et al., 2017 \\
\hline & Alkylating agent & Cyclophosphamide & Alexander et al., 2017 \\
\hline \multirow[t]{2}{*}{ Cardiology } & Statin & Lovastatin & Yoo et al., 2014 \\
\hline & Cardiac glycoside & Digoxin & Haiser et al., 2013 \\
\hline Diabetes & Biguanide & Metformin & Wu et al., 2017 \\
\hline Gastroenterology & Sulfa drug & Sulfasalazine & $\begin{array}{l}\text { Peppercorn and Goldman, } \\
1972 \mathrm{~b}\end{array}$ \\
\hline \multirow[t]{5}{*}{ Psychiatry } & Antipsychotic & Olanzapine & Davey et al., 2012 \\
\hline & & Risperidone & Bahr et al., 2015 \\
\hline & Benzodiazepine & Bromazepam & Fujii et al., 1987 \\
\hline & & Clonazepam & Elmer and Remmel, 1984 \\
\hline & & Nitrazepam & Takeno and Sakai, 1991 \\
\hline Neurology & Precursor & L-Dopa & Bergmark et al., 1972 \\
\hline
\end{tabular}

atherosclerosis in humans (Miller et al., 2014). There are still open questions as to what exactly constitutes a toxic level of TMAO, as even in the absence of dietary sources of methylamines, baseline levels of TMA and TMAO are found in the circulation (De La Huerga et al., 1953; Hoyles et al., 2018b). It is important to acknowledge that circulating TMAO may play a role in the protection from hyperammonemia (Kloiber et al., 1988) and from glutamate neurotoxicity (Minana et al., 1996). Chronic exposure of mice to TMAO attenuates diet-associated glucose tolerance, reducing endoplasmic stress and adipogenesis in adipocytes (Dumas et al., 2017). Flavin monooxygenase 3 , associated with the conversion of TMA to TMAO, is downregulated by testosterone (Bennett et al., 2013), suggesting possible differential processing of TMA in men and women.

In the context of inflammatory disease, the microbiota is responsible for the conversion of the prodrug sulfasalazine into 5-aminosalicylate (5-ASA), the active agent with anti-inflammatory properties used in the treatment of IBD (Peppercorn and Goldman, 1972b). This is a reaction carried out by microbial azoreductases, enzymes noted to be present in a wide variety of anaerobic bacteria from the human microbiota (Chung et al., 1992), making it difficult to envisage a loss of this function being associated with the gut microbiome alterations noted in IBD and resonating with the concept of functional resilience mentioned earlier. It is, however, notable that 5-ASA can be converted to $\mathrm{N}$-acetyl 5-ASA by intestinal bacteria, a reaction that does show variability in human fecal samples (van Hogezand et al., 1992). The metabolism of azo dyes to aromatic amines is also carried out by azoreductases, and fibers, antibiotics, or supplementation with live cultures of lactobacilli were able to affect azoreductase enzyme activity (Chung et al., 1992), an interesting observation in light of their potential application in IBD.

Drugs used in the treatment of cancer also represent examples of microbially-processed therapies and may be associated with adverse and toxic side effects, as is the case with irinotecan (Wallace et al., 2010). This reaction involves $\beta$-glucuronidases, bacterial enzymes noted to have a wide distribution in the gut microbiome (Cole et al., 1985). It has also been demonstrated more generally that of a panel of 30 chemotherapeutic drugs examined in vitro against various bacteria, 10 were found to be inhibited and six exhibited improved efficacy in a way that could be linked to modification of the chemical structure of the drug in the case of gemcitabine, fludarabine, cladribine, and CB1954 (Lehouritis et al., 2015).

Drug-drug interactions can also arise as a consequence of microbial biotransformations. The hydrolysis of the antiviral drug sorivudine to bromovinyluracil, which is then subsequently metabolized by the host to an inhibitor of host dihydropyrimidine dehydrogenase, may lead to toxicity due to inhibited degradation and increased concentrations of coadministered 5-fluorouracil (5-FU) (Nakayama et al., 1997). Specifically, conversion of bromovinyluracil by host dihydropyrimidine dehydrogenase produces a reactive intermediate that irreversibly inhibits the enzyme (Nishiyama et al., 2000). The production of bromovinyluracil is carried out by bacterial phosphorolytic enzymes with high enzymatic activity observed in Bacteroides species (Alexander et al., 2017).

More recently it has been demonstrated that the widely present bacterial uracil phosphoribosyltransferase (Martinussen and Hammer, 1994) can convert 5-FU to the RNA-damaging 5-fluorouridine monophosphate using the nematode Caenorhabditis elegans as a model system (Garcia-Gonzalez et al., 2017; Scott et al., 2017). Also using this model, it has been demonstrated that the deglycosylation of doxorubicin by Raoultella planticola is associated with reduced toxicity (Yan et al., 2018). Other gut-microbiota-mediated mechanisms through which the pharmacological effects of chemotherapeutics and immune therapeutics can be modulated include translocation and immunomodulation following interventions such as cyclophosphamide, doxorubicin, and 
anti-CLTA-4 therapies (Alexander et al., 2017). Understanding how alterations in gut microbiota profiles can dictate the host response to chemotherapeutic drugs may have important implications in clinical practice. Indeed, recent studies confirm the potential importance of patient stratification on the basis of gut microbiome composition to identify responders and nonresponders to immunotherapy for the treatment of epithelial tumors and melanomas (Gopalakrishnan et al., 2018; Jobin, 2018; Routy et al., 2018) (Fig. 5).

\section{B. Impact of Current and Putative Therapeutic Interventions on Microbial Community Structure and Function}

Many xenobiotics also impact on the composition of the gut microbiome, often in a clinically meaningful way. The possible extent of these occurrences was revealed recently following a screen of the interactions between over 1000 marketed and individual gut bacteria (Maier et al., 2018). The authors reported that $24 \%$ of the drugs with human targets across therapeutic classes inhibited the growth of at least one bacterial strain in vitro. Commonly used drugs, such as antipsychotics, proton-pump inhibitors (PPIs), hormones, and anticancer drugs, were included in the screen. Although the study largely focused on the in vitro antibacterial activity of each drug screened against one representative bacterial strain per species, the implications for more complex bacterial communities need to be considered. The results are also consistent with reported interactions between these drugs (antipsychotics, PPIs, and anticancer drugs) and the gut microbiome documented in previous preclinical and clinical studies (Davey et al., 2012, 2013; Imhann et al., 2016; Gopalakrishnan et al., 2018).

Exposure in incubation experiments to a panel of hosttargeted drugs, including digoxin and sulfasalazine, induces subtle changes in community structure, but more marked changes in the genes expressed by the gut microbiome, including those genes involved in xenobiotic metabolism (Maurice et al., 2013). Medication use was also confirmed to be an important

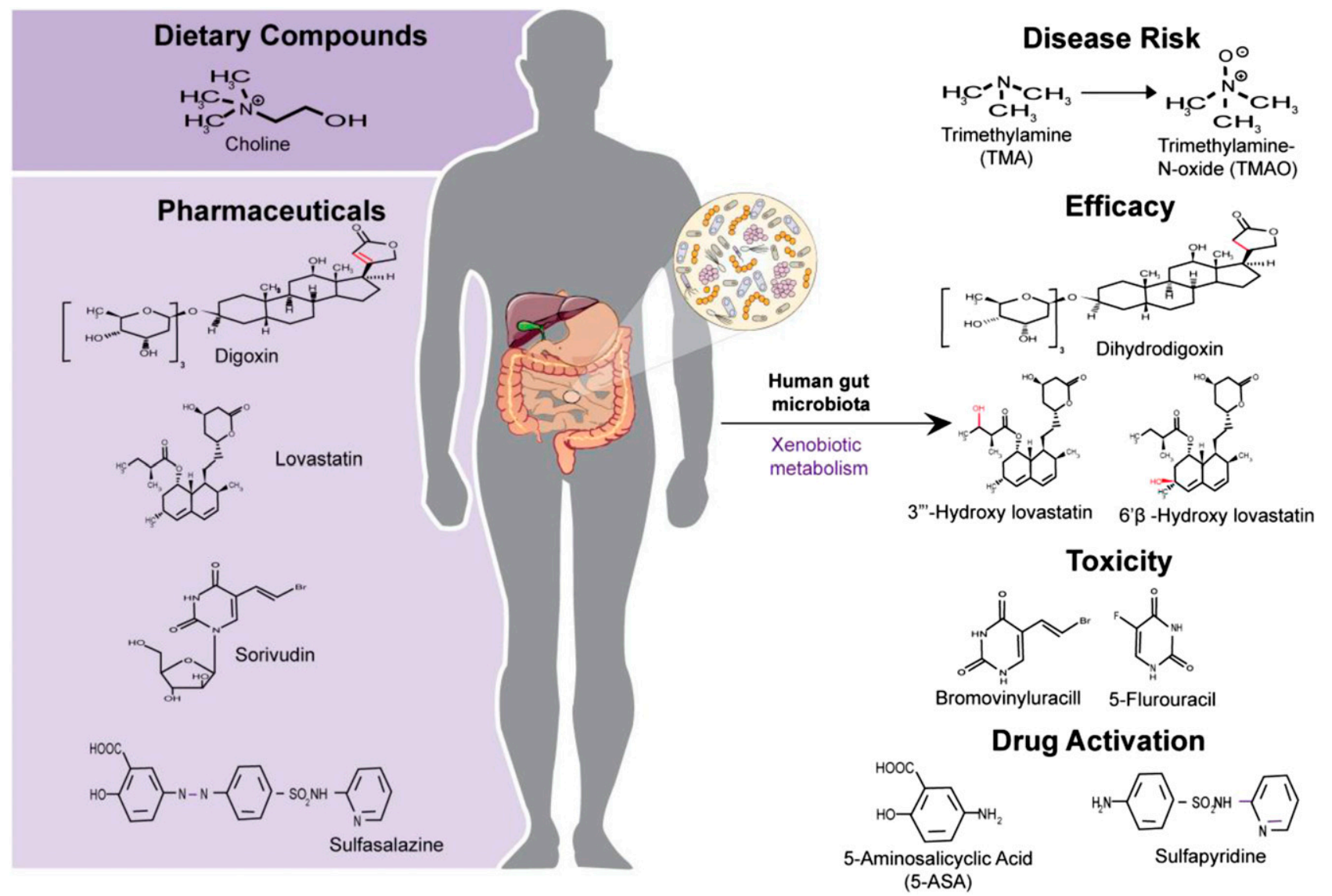

Fig. 5. Examples of clinically relevant microbial biotransformations. Direct microbial biotransformation of xenobiotics occurs following their ingestion, and, once present in the intestinal lumen, an array of bacterial enzymes is capable of modifying a diverse set of chemical structures, including dietary and pharmaceutical compounds. Taken together, this functional metabolic repertoire includes the capacity for clinically relevant activation (e.g., sulfasalazine) or inactivation (digoxin). Microbiota-drug interactions of relevance to cardiovascular pharmacology potentially include the hydroxylation of lovastatin. Drug-drug interactions can also arise as a consequence of microbial biotransformations, such as the hydrolysis of the antiviral drug sorivudine to bromovinyluracil. Bacterial conversion of dietary choline to TMA can lead to artherosclerosis as a consequence of further metabolism by host hepatic processing to TMAO. The consequences of microbial metabolism are thus of broad importance for disease risk, and drug efficacy and toxicity. 
covariate associated with human microbiota compositional variation in a recent large-scale study of Belgian and Dutch populations (Falony et al., 2016). A number of studies focused on specific candidates have also shone a light on this important area, as discussed below.

Metformin, used for the treatment of type 2 diabetes, has recently been shown to alter the gut microbiota composition of treatment-naive individuals in a way that improves glucose tolerance. This effect was probably achieved by promoting functional shifts in the gut microbiome with, for example, the enrichment of genes for carbohydrate metabolism, amino acid metabolism, and lipopolysaccharide biosynthesis (Wu et al., 2017). Metformin appears to have a dose-dependent antimicrobial effect with inhibition of specific bacterial strains only apparent once tested at physiologic intestinal concentrations (Maier et al., 2018). Interestingly, metformin can also promote in vivo the growth of specific bacterial species such as Akkermansia muciniphila and Clostridium cocleatum (Lee and Ko, 2014). This of course makes it difficult to distinguish type 2 diabetes versus metformin treatment signatures when assessing human gut microbiota composition (Forslund et al., 2015). Irinotecan can also alter the composition of the intestinal microbiome in rats, although it is unclear how well this aligns with the toxicity of this treatment (Lin et al., 2012; Alexander et al., 2017).

Methotrexate results in reduced microbiota diversity and compositional shifts in the abundance of Bacteroides associated with the induction of diarrhea in rats (Fijlstra et al., 2015). A recent systematic review indicates that other prescription medications are also associated with alterations in gut microbiome diversity, including PPIs, opioids, and NSAIDs (Le Bastard et al., 2018). The effect of PPIs in particular on gut microbiota diversity has been noted to be characteristic of an unhealthy gut microbiome and may increase the risk for enteric infections (Imhann et al., 2016).

There is mounting interest in the possibility that psychotropic agents might alter the composition of the gut microbiome (Kelly et al., 2016b). Currently, this has best been explored for the atypical antipsychotic drug olanzapine, which induces alterations in gut microbiome composition in rodents linked to the weight gain and other metabolic side effects that are associated with its use (Davey et al., 2012, 2013; Morgan et al., 2014). It has also been demonstrated that olanzapine has antimicrobial properties in vitro against two bacterial strains commonly residing in the human gut (Morgan et al., 2014). It is of note that olanzapine is metabolized by hepatic glucuronidation, and an unusually high percentage (30\%) of an oral dose is recoverable in human feces (Kassahun et al., 1997). Prebiotic administration concurrently with olanzapine can reduce the antipsychoticinduced weight gain in rodents (Kao et al., 2018). Another antipsychotic, risperidone, also causes shifts in gut microbiome composition in mice (Bahra et al., 2015), and in children and adolescents (Bahr et al., 2015). More recently, it has been demonstrated that similar bacterial species are affected by different and chemically diverse antipsychotics (Maier et al., 2018). Chronic alcohol abuse also alters gut microbiome composition (Leclercq et al., 2017), and other drugs of abuse possibly do the same, such as methamphetamine (Ning et al., 2017). More generally, substance use disorders are associated with reduced microbiota diversity (Xu et al., 2017).

Although the therapeutic potential of microRNAs (miRNAs) is far from fully realized, these small nucleotide sequences with the ability to regulate gene expression represent molecular effectors of importance in a range of areas, including psychiatric disorders such as depression and anxiety (Scott et al., 2015; Gururajan et al., 2016; O'Connor et al., 2016) as well as cancer (Slaby et al., 2017) and cardiovascular disorders (Mellis and Caporali, 2018). Understanding the role of the gut microbiome-miRNA interactions is a novel departure that has seen evidence emerge from studies in germ-free rodents of microbial regulation of miRNA expression in brain regions of importance for neuropsychiatry such as the amygdala, prefrontal cortex, and hippocampus (Chen et al., 2017; Hoban et al., 2017; Moloney et al., 2017). It also seems that host miRNA production by intestinal epithelial cells has a role in shaping the gut microbiome by affecting bacterial growth (Liu et al., 2016), and fluctuations in microbial composition of the gut microbiome are associated with alterations in fecal miRNAs (Moloney et al., 2018). This will be an important consideration if the miRNA-targeted therapeutics in clinical development make their way to the clinic for the treatment of disorders associated with gut microbiome alterations.

Dietary compounds exert a major impact on the gut microbiome. In mice, a number of nutritional and nutraceutical interventions for cardiovascular disease were compared against a standard pharmaceutical intervention for their impact on the gut microbiome. Plant sterol ester produced the strongest compositional effect with more modest alterations noted following oat $\beta$-glucan and bile salt hydrolase-active $L$. reuteri APC 2587, with few effects noted for atorvastatin. Functional alterations following plant sterol ester, oat $\beta$-glucan, and atorvastatin were also noted in this study, using microbial and host-derived metabolites in the serum metabolome as a readout (Ryan et al., 2017). Polyunsaturated fatty acids (PUFAs) are thought to exert an antidepressant effect, possible via their antiinflammatory properties (Burhani and Rasenick, 2017). Omega-3 PUFAs also have important cardioprotective and neurodevelopmental properties and support the function and aging of the central nervous system (Pusceddu et al., 2016). Dietary omega-3 PUFA status impacts on the cecal microbiome and metabolome in 
mice (Robertson et al., 2017b) and is also able to impact on gut microbiota development in adolescence and adulthood when varied in the maternal and earlypostnatal diet (Robertson et al., 2017a). It has also been reported that the disruptions to the gut microbiota induced by the early-life stressor maternal separation in female rats can be rescued by omega-3 PUFA supplementation (Pusceddu et al., 2015). In clinical populations, and although omega-3 PUFA supplementation did not change gut microbiome diversity or composition at the phyla level, an increase in several short chain fatty acidproducing bacteria such as Bifidobacterium, Roseburia, and Lactobacillus was noted (Watson et al., 2017).

Dietary phytochemicals can induce gut microbiota alterations, exerting prebiotic-like effects to stimulate the growth of beneficial bacteria (Laparra and Sanz, 2010). Recent revisions of the definition of prebiotics have expanded their scope and now include both PUFAs and phytochemicals (Gibson et al., 2017). Polyphenolic phytochemicals in particular are known to exert beneficial health effects beyond their antioxidant capacity (Stevenson and Hurst, 2007). Recent evidence suggests that resveratrol, for example, may act to remodel the gut microbiome to yield cardiovascular benefits, antiobesity effects, and improvements in glucose homeostasis (Chen et al., 2016; Bird et al., 2017; Sung et al., 2017). Flavonoids also shape gut microbiota membership and function (Oteiza et al., 2018). Other dietary compounds known to alter the composition of the gut microbiome include noncaloric artificial sweeteners such as saccharin, sucralose, and aspartame (Suez et al., 2015), a feature with functional implications associated with the induction of glucose intolerance in mice (Suez et al., 2014). Although probiotics are not thought to substantially change the global compositional characteristics of the gut microbiome (Kristensen et al., 2016), they do impact on functional metabolic outputs such as the production of short chain fatty acids and other pharmabiotics, which may be important in their propensity to influence host health (Patterson et al., 2014).

\section{Environmental and Industrial Chemicals}

The capacity of the gut microbiome to metabolize environmental chemicals is mediated by enzymes with a wide distribution such as $\beta$-glucoronidases, azoreductases, and nitroreductases, which are able to chemically transform a range of agents such as nitrotoluenes, pesticides, polychlorobiphenyls, azo dyes, and metals (Claus et al., 2016). Many of these same chemical agents can also alter the composition and/or function of the gut microbiota. Chronic exposure to low doses of the insecticide, Chlorpyrifos, changed the composition of the gut microbiota in both a simulated human intestinal microbiota preparation and rats, reducing the abundance of Lactobacillus spp. and Bifidobacterium spp, bacteria presumed to be beneficial (Joly et al., 2013).
Glyphosate, the most commonly used herbicide (Cuhra et al., 2016), inhibits microbial enzymes (5enolpyruvylshikimate-3-phosphate synthase), and some glyphosate formulations do have antibacterial properties (Tarazona et al., 2017). It remains to be seen whether the detrimental impact of glyphosate seen on soil, plant, and farm animal microbiomes will be consistently reflected in the human microbiome where the concentrations reached are usually much lower (Flandroy et al., 2018). Heavy metals such as mercury, lead, cadmium, and arsenic may also have an important impact on the gut microbiota (Lu et al., 2015). Traffic-related air pollution can result in a decreased relative abundance of Bacteroidaceae and an increased relative abundance of Coriobacteriaceae (Alderete et al., 2018). Exposure to endocrine-disrupting chemicals such as bisphenol A and ethinyl estradiol, by incorporation into the diet from periconception through weaning, induced generational and sex-dependent gut microbiome changes in mice, including increased relative abundances of Bacteroides, Prevotellaceae, and Akkermansia (Javurek et al., 2016). The collective literature in this area requires further elaboration to understand the implications for host health, not least within a framework that places the characteristics of the early-life microbiome as critical to the subsequent emergence of adult pathologies (Clarke et al., 2014a; Claus et al., 2016) (Fig. 6).

\section{Experimental Approaches in Pharmacomicrobiomics}

Understanding the complex and bidirectional interplay between the gut microbiome and xenobiotics requires an array of experimental approaches geared toward extracting the necessary complementary information. Many of the strategies employed, such as the use of microbiota-deficient animals, are commonly applied across cognate areas of microbiome research (Williams, 2014; Luczynski et al., 2016). This includes both germ-free models or those generated via depletion of the gut microbiota with a cocktail of antibiotics, and can provide valuable pharmacokinetic and pharmacodynamic insights. These approaches are not without their limitations, not least because of the intrinsic gastrointestinal and microbial differences between humans and rodents (Nguyen et al., 2015). There are also physiologic and morphologic differences between germ-free animals and conventional controls relevant for xenobiotic metabolism (Sousa et al., 2008). For example, host hepatic enzyme expression and activity are altered in animals reared in a germ-free environment (Selwyn et al., 2016). However, the use of these animal models in monoassociation studies or following the transplant of human microbiota profiles is clearly an invaluable option (Haiser et al., 2014). Insights from invertebrate models, such as the nematode C. elegans, are 


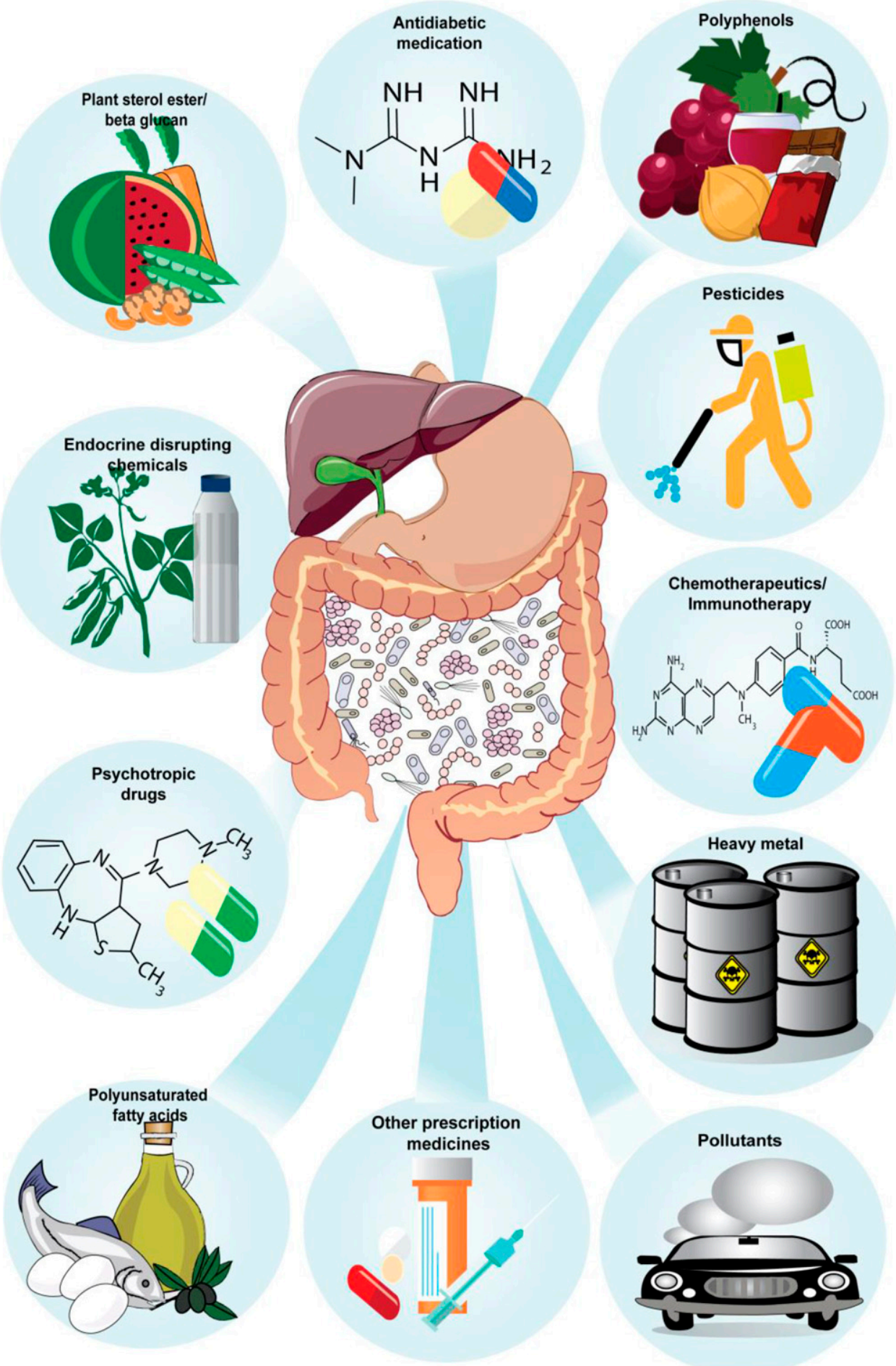

Fig. 6. Xenobiotics that alter the gut microbiome. Many xenobiotics also impact on gut microbiome characteristics. This includes pharmaceutical compounds such as metformin and methotrexate and psychotropic agents such as olanzapine. Other prescription medications are associated with alterations in gut microbiome diversity, including PPIs, opioids, and NSAIDs. Dietary compounds also exert a major impact on the gut microbiome, including polyphenolic phytochemicals (resveratrol, flavonoids), polyunsaturated fatty acids, and plant sterol ester. In addition, environmental and industrial chemicals, including endocrine disrupting chemicals, heavy metals, pesticides, and pollutants, exert an influence on gut microbiome structure, composition, and function.

increasingly used to inform our understanding of host-microbe interactions (Clark and Walker, 2018), and in the current context, a number of examples have been discussed, including studies focused on the chemotherapeutics doxorubicin (Yan et al., 2018) and 5-FU (Garcia-Gonzalez et al., 2017; Scott et al., 2017).

Conceptually, contrasting lower gut metabolites with upper gut metabolites in rodents or comparing extended 
release versus immediate release formulations or i.v. drug delivery with oral drug delivery in human in vivo studies can also be informative (Sousa et al., 2008). More routine screening of reciprocal microbiome-xenobiotic interactions can be conducted in various in vitro batch culture or fermentation systems or in the more complex simulator of the human intestinal microbial ecosystem, as was demonstrated for the insecticide Chlorpyrifos (Sousa et al., 2008; Joly et al., 2013; Ou et al., 2015). Large scale in vitro screening of commonly used drugs for antibacterial activity also offers important information (Maier et al., 2018). There are also advantages to the use of in silico models to predict outcome from a variety of inputs, possibly by combining microbiota metabolism rates with host drug absorption and metabolic rates. Specifically adapting many of the emerging physiologically based pharmacokinetic models, which predict drug levels in tissues based on a variety of drug and host parameters (Min and Bae, 2017; Thiele et al., 2017; Donovan et al., 2018), to allow for greater contribution of microbiota-mediated metabolism, may be a useful approach to predict overall clinical impact.

Fecal sample incubations are often used in xenobiotic metabolism studies, as they are easily accessible and provide a reasonably reliable means of assessing the metabolic activity of the colonic microbiota. Fecalase or cecalase, stable and translationally relevant cell-free extracts of feces or cecal matter with bacterial enzymatic activity, are frequently used incubations (Tamura et al., 1980). This has been applied in the study of lovastatin (Yoo et al., 2014) and amlodipine (Yoo et al., 2016) and to look at the effect of probiotics on the pharmacokinetics of acetaminophen (Kim et al., 2018). Concerns about the variability of the assay have seen the development of a fecal microbial enzyme mix (Yeo et al., 2012).

Haiser et al. (2014) have outlined how these approaches might be combined in a framework for studying microbial drug metabolism that includes functional metagenomics to gain mechanistic insights and bioinformatics to guide rational microbiome-targeted therapeutic interventions. The value of such an approach once it leads to well-defined enzyme biochemistry can be seen in an earlier study that demonstrated that it was possible to selectively inhibit bacterial $\beta$-glucuronidases to alleviate drug-induced gastrointestinal toxicity arising from irinotecan (Wallace et al., 2010). There is also a variety of predictive and computational tools for the evaluation of microbial effects on drugs during gastrointestinal passage that can be used to streamline the process and provide targets for downstream in vitro and in vivo hypothesis assessments (Pieper and Bertau, 2010; Klunemann et al., 2014; Magnúsdóttir and Thiele, 2018).

Many of the in vitro approaches discussed are perhaps overly simplistic and do not best capitalize on the unique features of the gut ecosystem, such as microniches, $\mathrm{pH}$ gradients, and dynamic microbe-tissue interactions (Tralau and Luch, 2013). Organ-on-a-chip microphysiological systems are likely to increase efficiency and contribute in the future to a better understanding of microbial metabolism and host-microbiome crosstalk (Park et al., 2017). Gastrointestinal organoids can be used to more accurately model aspects of epithelial barrier dynamics, including cellular differentiation and proliferation, in specific intestinal segments bring advantages over cell culture models in unraveling the molecular basis of the host-microbe interactions (Hill and Spence, 2016). Meanwhile, recent reports of an ingestible electronic capsule that can provide real-time information on gastrointestinal oxygen, hydrogen, and carbon dioxide may ultimately see use as a chemical biosensor to improve our understanding of the biogeography of gut microbial metabolism (Kalantar-Zadeh et al., 2018). Indeed, this is already partially functional in this regard because the detection of hydrogen signals can be used as a proxy for regional fermentation patterns (Fig. 7).

\section{Toward Microbiome-Based Treatments and Novel Biotherapeutics}

The prospect of targeting the microbiome for enhancing drug efficacy and therapeutic benefit is appealing, and the development of innovative, more personalized approaches in the practice of medicine can potentially be expedited with successful incorporation of our knowledge of hostmicrobe interactions outlined above. A number of other options are also currently under consideration and include drug delivery via designer probiotics or biotherapeutics that take advantage of the modified biochemical prowess of gut bacteria (Maxmen, 2017). For example, a genetically engineered Lactococcus lactis-secreting interleukin-10 has been used to deliver a localized therapeutic dose of IL-10 for the amelioration of murine colitis in an animal model of IBD (Steidler et al., 2000). The use of designer lactic acid bacteria as factories for the production of antimicrobial and anti-inflammatory biomolecules may also see utility in the future treatment of infectious diseases, cancer, and metabolic diseases (Cano-Garrido et al., 2015; Singh et al., 2017). Meanwhile, nextgeneration probiotics may be based on the use of microbes found to be deficient in certain disorders, as is the case of $A$. muciniphila in obesity, diabetes, and cardiometabolic diseases (Cani and de Vos, 2017), or Faecalibacterium prausnitzii in IBD if the positive preclinical results in models of colitis successfully translate to the clinic (O'Toole et al., 2017). Pathogen-specific antimicrobials and the development of bacteriocins as alternative therapeutic options is another avenue under exploration (Maxson and Mitchell, 2016; Mathur et al., 2017; Munguia and Nizet, 2017), as is the potential use of bacteriophage (Forde and Hill, 2017). Precision editing of the gut microbiota may also be possible, with an example being the use of tungstate treatment to selectively inhibit microbial respiratory pathways, which are operational specifically 


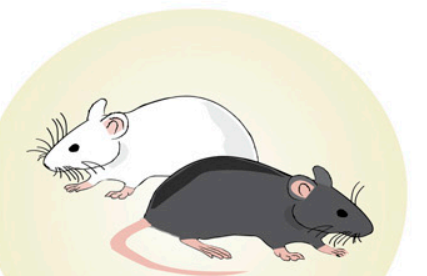

Microbiota-deficient animal model

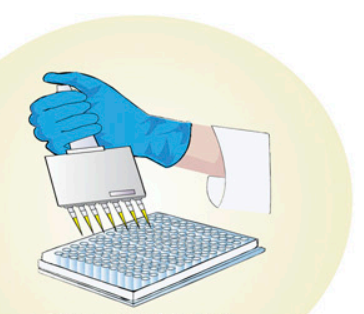

Pharmacokinetic assay

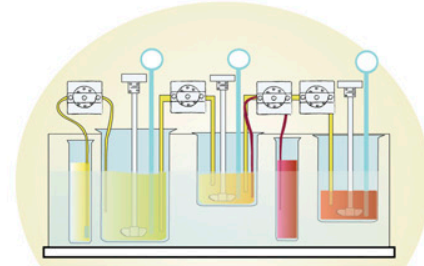

Simulator of the human intestinal microbial ecosystem (SHIME)

$\Delta$

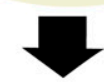

2

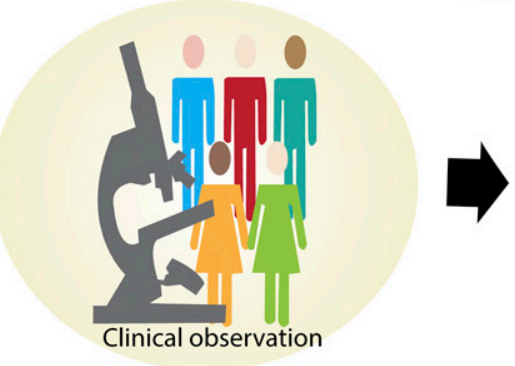

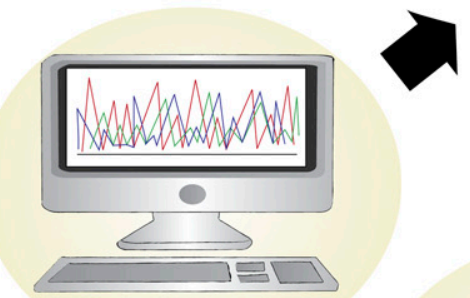

Bioinformatic prediction

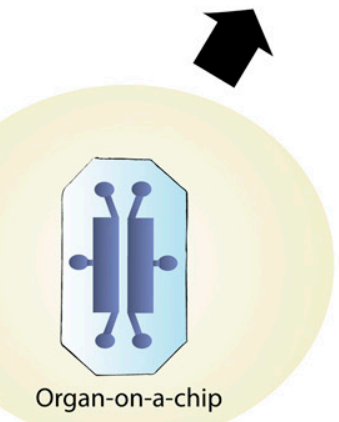

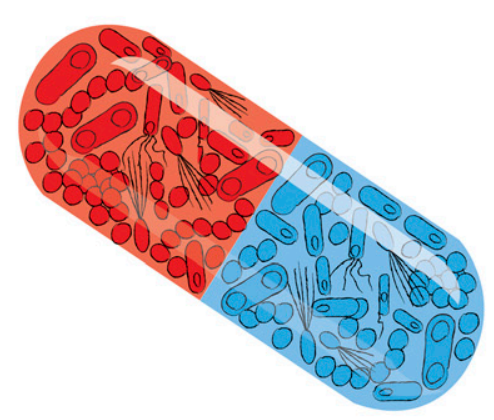
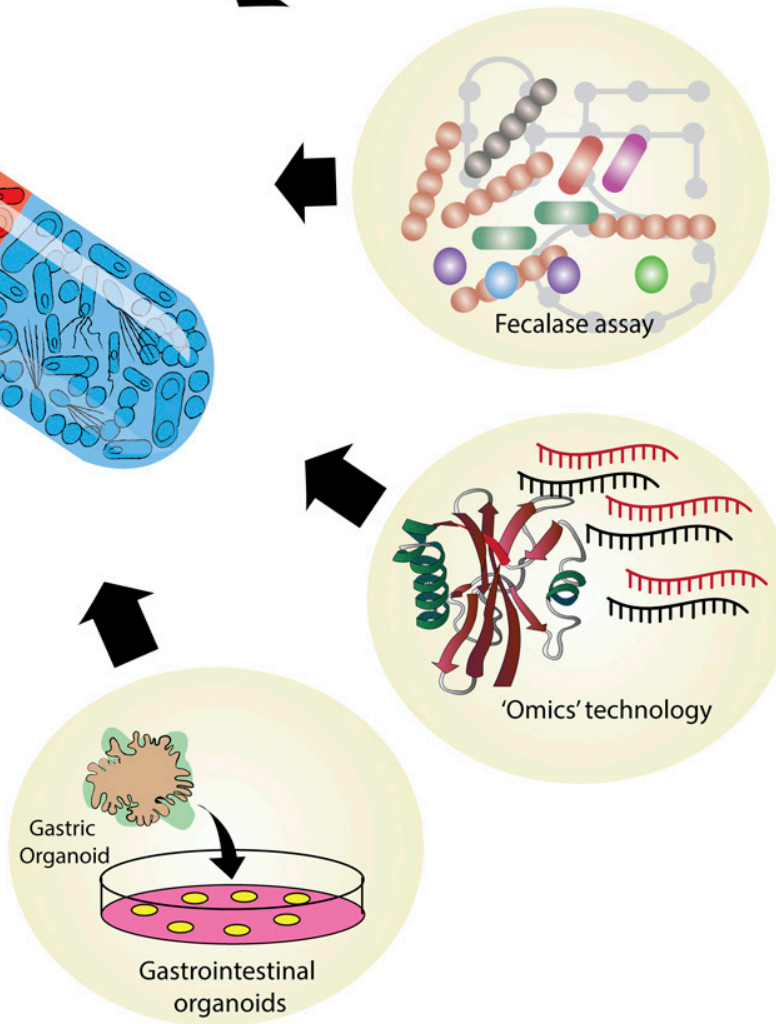

'Omics' technology

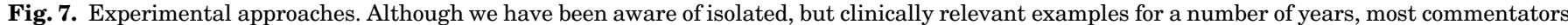

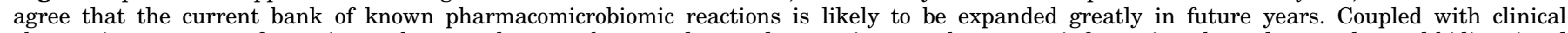

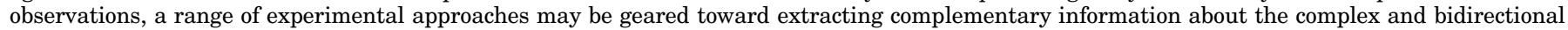

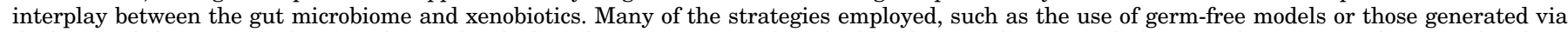

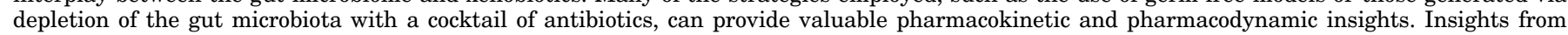

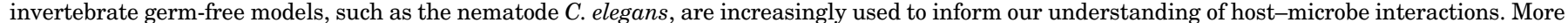

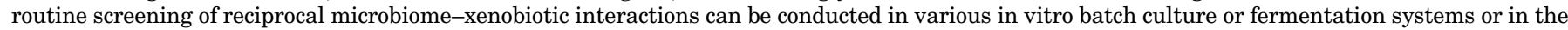

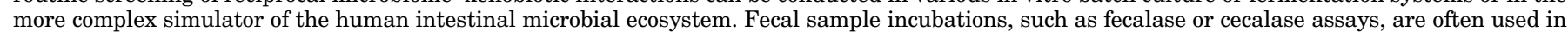

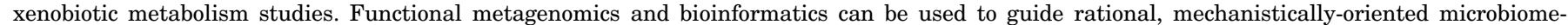

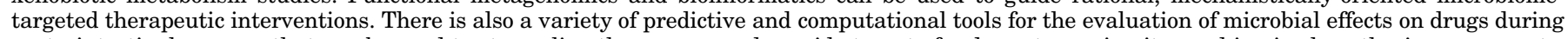

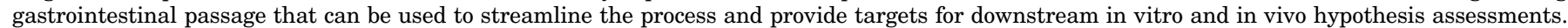

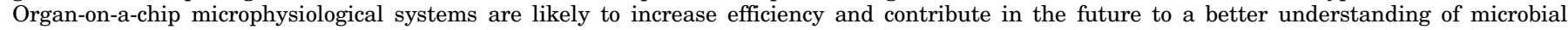

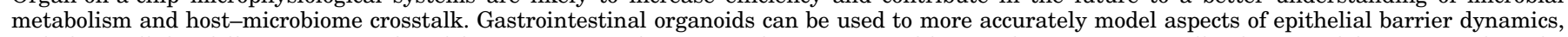

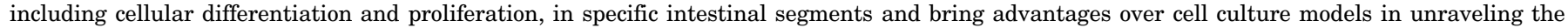
molecular basis of the host-microbe interactions.

during episodes of localized gut inflammation (Zhu et al., 2018). It remains to be seen whether fecal microbiota transplantation, which has been so effective in the treatment of recurrent Clostridium difficile infection, will also see applications in this area (de Groot et al., 2017).

\section{A. Psychobiotics}

Psychobiotics were originally defined as "a live organism that, when ingested in adequate amounts, produces a health benefit in patients suffering from psychiatric illness" (Dinan et al., 2013). That has since expanded to include prebiotics that enhance the growth of beneficial gut bacteria and also to include healthy individuals (Sarkar et al., 2016). Currently, preclinical behavioral screening platforms are most frequently used to identify candidate psychobiotics for further evaluation in clinical platforms (Bravo et al., 2011; Savignac et al., 2014, 2015; Burokas et al., 2017). This 
has met with somewhat mixed results in healthy human subjects with strain-specific positive results (Allen et al., 2016; Papalini et al., 2018) and a failure to translate (Kelly et al., 2017). Refinements of the discovery pipeline may be necessary at various pressure points, and this could include the development of psychobiotics with specific mechanisms of action, tailored toward individual patient requirements (Bambury et al., 2018).

One option in this regard may be the selection of psychobiotics to fine-tune host or microbial tryptophan metabolism, an important source of neuroactives for microbiome-gut-brain axis signaling, including therapeutic targets for stress-related disorders such as serotonin and kynurenine (O'Mahony et al., 2015; Yano et al.,
2015; Kennedy et al., 2017). Candidate psychobiotics that beneficially impact on host tryptophan metabolites include Bifidobacterium longum subsp. longum 35624 (previously B. infantis 35624) (Desbonnet et al., 2008) and $L$. reuteri (Marin et al., 2017). Bacterial metabolism of tryptophan to indole compounds may be equally important because either in their own right or following host processing, these microbial tryptophan metabolites can impact on host physiology and behavior (Lee et al., 2015). Next-generation psychobiotics may be selected to metabolize tryptophan at specific gut locations, as is the case for specific mucin utilizing Peptostreptococcus species, which produce indoleacrylic acid close to the intestinal epithelium, where it acts

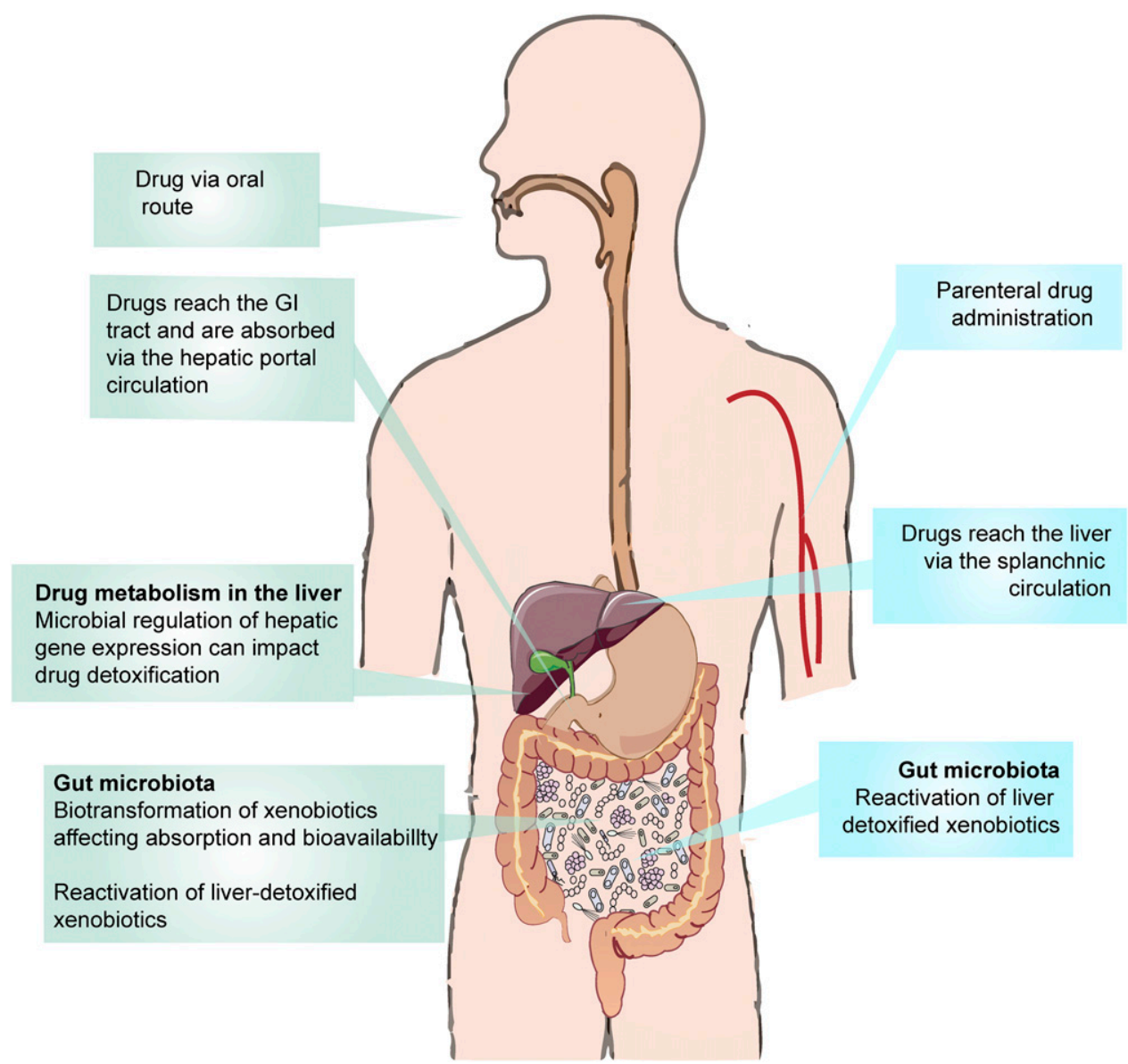

Fig. 8. Microbial contribution to drug metabolism. Following enteral administration, the physiochemical properties or formulation of a drug impacts the region of the gastrointestinal tract where the major exposure to microbial drug metabolism occurs. The nature of these interactions depends on whether the compounds encountered by the gut microbiome are poorly absorbed or are first conjugated by the liver and subsequently reach the intestine via biliary excretion, which is also a route through which i.v. administered drugs can become subject to chemical transformation by gut microbes. The majority of orally administered drugs are normally absorbed in the upper small intestine assuming that the drug is highly soluble and highly permeable in this region. Although there is a lower density of microbes in the small intestine, we have an incomplete understanding of the impact of microbial activity in different regions of the gastrointestinal tract, and there may be important implications of region-specific microbial drug metabolism for health and disease. However, many drugs display poor solubility, which can lead to slow and incomplete absorption with the drug being absorbed from distal regions of the small intestine and/or the colon. Drugs with low permeability through the intestinal membrane can lead to greater amounts of those drugs reaching these distal regions of the intestine. Drugs can also become a candidate substrate for direct microbial processing if they are appropriately formulated. The highest density of bacteria is found in the colon, and the prevalent use of advanced drug formulations designed for extended release (e.g., sustained release tablets) is on the increase, circumventing absorption profiles that would usually limit exposure of many drugs to the colonic gut microbiota. This ensures that an increasing number of pharmaceuticals are first exposed to the gut microbiota and their associated collection of metabolic enzymes with the potential for relevant microbiome-drug interactions highest in this region. The fate of drugs following oral ingestion is most often considered, but parenteral routes can still lead to gut microbiome-drug interactions, for example, via the splanchnic circulation and bilary excretion. Enterohepatic circulation is an important consideration in this regard, as is the potential for an indirect impact via microbial regulation of hepatic drug metabolism. 
to improve barrier function (Wlodarska et al., 2017). Gut-derived microbial metabolites such as propionate, whose production could be stimulated by prebiotics, can also exert protective effects on blood-brain barrier integrity (Hoyles et al., 2018c). Given the importance of intestinal and blood-brain barrier permeability for health and disease (Bischoff et al., 2014; Kelly et al., 2015), this strategy could be combined with the selection of at-risk or compromised individuals to improve treatment outcomes.

\section{Summary, Conclusions, and Perspectives}

The gut microbiome can have a wide variety of direct and indirect effects on the metabolism of pharmaceuticals, dietary compounds, and environmental chemicals. The pharmacomicrobiomic web portal currently lists over 90 examples of such chemicals (http://pharmacomicrobiomics. $\mathrm{com} / \mathrm{view} / \mathrm{chemical} /$ ). Although we have been aware of isolated, but clinically relevant examples for a number of years, most commentators agree that the current bank of known reactions is likely to be expanded greatly in future years. For drug metabolism alone, the implications of microbial biotransformation or other indirect actions of gut bacteria are hugely significant because it results in the activation or inactivation of drugs and often generates byproducts that are toxic to the host (Fig. 8).

An increased focus on this important area comes at a time when sequencing studies have identified gut microbiome alterations in many of the disorders treated by these same drugs. The reciprocal nature of these microbe-drug interactions has also been demonstrated with many of these therapeutic agents also modifying the composition and function of the gut microbiome (see Table 3), although increased application of techniques to study both microbial transcriptional activities and metabolic profiles is warranted (Carmody and Turnbaugh, 2014). Taken together, this paints a complex picture, and whereas a research framework is being put in place, cross-disciplinary input and increased attention from the scientific community are required to address the many outstanding questions in this field (see Table 4) and to achieve a more comprehensive view of pharmacology (Saad et al., 2012; Carmody and Turnbaugh, 2014). We can take encouragement in these efforts from recent observations regarding the importance of gut microbiome composition and the response to cancer therapeutic drugs (Jobin, 2018). If this can even be partially replicated across the range of disorders discussed above, the move toward the practice of precision, personalized medicine may well be expedited by incorporation of microbiome research.

Although there are many examples of remediation opportunities, we should not neglect the prophylactic power of diet to help sculpt the diversity that is necessary in a healthy gut microbiome (Shanahan et al., 2017). We have noted above a number of instances in which diet-microbe-host interactions can lead to adverse consequences. Indeed, the prescription of dietary modifications is also now being considered in a number of disease areas, including psychiatry (Jacka, 2017), as are other lifestyle factors such as exercise, which is also known to improve gut microbiota profiles (O'Sullivan et al., 2015). Stress exposure is also an important consideration, particularly during the extremes of life when the gut microbiota is more chaotic and vulnerable. Although there are many reports of elevated or decreased circulating levels of microbiomeassociated metabolites in pathologic states, there remains an incomplete understanding of their functional important in the absence of disease. Defining normal physiologic ranges and toxic concentrations is an important objective before interventions designed to remove specific bacteria and/or their associated functions from the human gut microbiome can be effectively implemented. Neither should we ignore the other human microbial ecological niches. For example, although we have focused above on the gut microbiome, microbicide efficacy in African women has been linked to vaginal microbiome characteristics, with tenofovir being depleted by bacterial metabolism (Klatt et al., 2017). Also neglected to date is the gut mycobiome, and there are indications that our intestinal fungi are an important component of host-microbe interactions of relevance to immune homeostasis and inflammatory disorders (Leonardi et al., 2018) and stress-related disorders such as irritable bowel syndrome (Botschuijver et al., 2017).

As our knowledge of the gut microbiome continues to increase and the balance shifts from new discoveries to mechanistic insights, so too does our appreciation that virtually all aspects of host physiology need to be

TABLE 4

Outstanding questions in pharmacomicrobiomics

Key Questions

\footnotetext{
Do disease-associated microbiomes impact on bacterial enzyme expression and/or activity to dysregulate xenobiotic metabolism?

What is the impact of extreme of life microbiome variation on pharmacokinetics and pharmacodynamics?

Can we unravel disease vs. drug-induced contributions to microbiome alterations?

Can the use of microbiome composition to predict therapeutic response be incorporated into clinical practice?

Can we identify bacteria- and/or enzyme-specific host-microbe interactions for subsequent therapeutic targeting?

Can we compile and systematically evaluate currently unexplained aberrant pharmacokinetic observations?

Can diet be used to beneficially tune microbial xenobiotic metabolism?

Can we differentiate between effects of acute and long-term exposure to xenobiotics on the gut microbiome?

Should we re-evaluate the guidelines for coadministration of antibiotics?
} 
reconsidered in light of reciprocal host-microbiome interactions. It is only now logical to include the broad metabolic capacity of the gut microbiome within fundamental principles of pharmacokinetics and pharmacodynamics, the difficulty in rationally manipulating the chemical biotransformations by unknown enzymes and members of the gut microbiome notwithstanding. The future practice of precision medicine may depend on our ability to successfully navigate the challenges that lie ahead and incorporate a more microbial perspective into clinical guidelines and applications.

\section{Acknowledgments}

We thank Sofia Cussotto for helpful comments on content and general discussions on this topic.

\section{Authorship Contributions}

Participated in research design: Clarke, Sandhu, Griffin, Dinan, Cryan, Hyland.

Performed data analysis: Clarke, Sandhu, Griffin, Dinan, Cryan, Hyland.

Wrote or contributed to the writing of the manuscript: Clarke, Sandhu, Griffin, Dinan, Cryan, Hyland.

\section{References}

Albenberg L, Esipova TV, Judge CP, Bittinger K, Chen J, Laughlin A, Grunberg S, Baldassano RN, Lewis JD, Li H, et al. (2014) Correlation between intraluminal oxygen gradient and radial partitioning of intestinal microbiota. Gastroenterology 147:1055-1063.e8.

Alderete TL, Jones RB, Chen Z, Kim JS, Habre R, Lurmann F, Gilliland FD, and Goran MI (2018) Exposure to traffic-related air pollution and the composition of the gut microbiota in overweight and obese adolescents. Environ Res 161: $472-478$.

Alexander JL, Wilson ID, Teare J, Marchesi JR, Nicholson JK, and Kinross JM (2017) Gut microbiota modulation of chemotherapy efficacy and toxicity. Nat Rev Gastroenterol Hepatol 14:356-365.

Allen AP, Curran EA, Duggan Á, Cryan JF, Chorcoráin AN, Dinan TG, Molloy DW, Kearney PM, and Clarke G (2017) A systematic review of the psychobiological burden of informal caregiving for patients with dementia: focus on cognitive and biological markers of chronic stress. Neurosci Biobehav Rev 73:123-164

Allen AP, Hutch W, Borre YE, Kennedy PJ, Temko A, Boylan G, Murphy E, Cryan JF, Dinan TG, and Clarke G (2016) Bifidobacterium longum 1714 as a translational psychobiotic: modulation of stress, electrophysiology and neurocognition in healthy volunteers. Transl Psychiatry 6:e939.

Andrade C (2015) Sustained-release, extended-release, and other time-release formulations in neuropsychiatry. J Clin Psychiatry 76:e995-e999.

Asano Y, Hiramoto T, Nishino R, Aiba Y, Kimura T, Yoshihara K, Koga Y, and Sudo $\mathrm{N}$ (2012) Critical role of gut microbiota in the production of biologically active, free catecholamines in the gut lumen of mice. Am J Physiol Gastrointest Liver Physiol 303:G1288-G1295.

Avilés-Jiménez F, Yu G, Torres-Poveda K, Madrid-Marina V, and Torres J (2017) On the search to elucidate the role of microbiota in the genesis of cancer: the cases of gastrointestinal and cervical cancer. Arch Med Res 48:754-765.

Bahr SM, Tyler BC, Wooldridge N, Butcher BD, Burns TL, Teesch LM, Oltman CL, Azcarate-Peril MA, Kirby JR, and Calarge CA (2015) Use of the second-generation antipsychotic, risperidone, and secondary weight gain are associated with an altered gut microbiota in children. Transl Psychiatry 5:e652.

Bahra SM, Weidemann BJ, Castro AN, Walsh JW, deLeon O, Burnett CM, Pearson NA, Murry DJ, Grobe JL, and Kirby JR (2015) Risperidone-induced weight gain is mediated through shifts in the gut microbiome and suppression of energy expenditure. EBioMedicine 2:1725-1734.

Bambury A, Sandhu K, Cryan JF, and Dinan TG (2018) Finding the needle in the haystack: systematic identification of psychobiotics. Br J Pharmacol 175:4430-4438.

Barton W, Penney NC, Cronin O, Garcia-Perez I, Molloy MG, Holmes E, Shanahan F, Cotter PD, and O'Sullivan O (2018) The microbiome of professional athletes differs from that of more sedentary subjects in composition and particularly at the functional metabolic level. Gut 67:625-633.

Benet LZ and Cummins CL (2001) The drug efflux-metabolism alliance: biochemical aspects. Adv Drug Deliv Rev 50 (Suppl 1):S3-S11.

Bennett BJ, de Aguiar Vallim TQ, Wang Z, Shih DM, Meng Y, Gregory J, Allayee H, Lee R, Graham M, Crooke R, et al. (2013) Trimethylamine-N-oxide, a metabolite associated with atherosclerosis, exhibits complex genetic and dietary regulation. Cell Metab 17:49-60.

Bergmark J, Carlsson A, Granerus AK, Jagenburg R, Magnusson T, and Svanborg A (1972) Decarboxylation of orally administered L-dopa in the human digestive tract. Naunyn Schmiedebergs Arch Pharmacol 272:437-440.
Bharwani A, Mian MF, Foster JA, Surette MG, Bienenstock J, and Forsythe P (2016) Structural \& functional consequences of chronic psychosocial stress on the microbiome \& host. Psychoneuroendocrinology 63:217-227.

Bird JK, Raederstorff D, Weber P, and Steinert RE (2017) Cardiovascular and antiobesity effects of resveratrol mediated through the gut microbiota. Adv Nutr $\mathbf{8}$ 839-849.

Bischoff SC, Barbara G, Buurman W, Ockhuizen T, Schulzke JD, Serino M, Tilg H, Watson A, and Wells JM (2014) Intestinal permeability--a new target for disease prevention and therapy. BMC Gastroenterol 14:189.

Blaser MJ (2016) Antibiotic use and its consequences for the normal microbiome. Science 352:544-545.

Bokulich NA, Chung J, Battaglia T, Henderson N, Jay M, Li H, D Lieber A, Wu F, Perez-Perez GI, Chen Y, et al. (2016) Antibiotics, birth mode, and diet shape microbiome maturation during early life. Sci Transl Med 8:343ra82.

Bolhuis MS, Panday PN, Pranger AD, Kosterink JG, and Alffenaar JW (2011) Pharmacokinetic drug interactions of antimicrobial drugs: a systematic review on oxazolidinones, rifamycines, macrolides, fluoroquinolones, and Beta-lactams. Pharmaceutics 3:865-913.

Bordenstein SR and Theis KR (2015) Host biology in light of the microbiome: ten principles of holobionts and hologenomes. PLoS Biol 13:e1002226.

Botschuijver S, Roeselers G, Levin E, Jonkers DM, Welting O, Heinsbroek SEM, de Weerd HH, Boekhout T, Fornai M, Masclee AA, et al. (2017) Intestinal fungal dysbiosis is associated with visceral hypersensitivity in patients with irritable bowel syndrome and rats. Gastroenterology 153:1026-1039.

Bravo JA, Forsythe P, Chew MV, Escaravage E, Savignac HM, Dinan TG, Bienenstock J, and Cryan JF (2011) Ingestion of Lactobacillus strain regulates emotional behavior and central GABA receptor expression in a mouse via the vagus nerve. Proc Natl Acad Sci USA 108:16050-16055.

Bright TV, Clark BR, O'Brien E, and Murphy CD (2011) Bacterial production of hydroxylated and amidated metabolites of flurbiprofen. J Mol Catal B Enzym 72: 116-121.

Burhani MD and Rasenick MM (2017) Fish oil and depression: the skinny on fats. $J$ Integr Neurosci 16:S115-S124.

Burokas A, Arboleya S, Moloney RD, Peterson VL, Murphy K, Clarke G, Stanton C, Dinan TG, and Cryan JF (2017) Targeting the microbiota-gut-brain axis: prebiotics have anxiolytic and antidepressant-like effects and reverse the impact of chronic stress in mice. Biol Psychiatry 82:472-487.

Campbell SC and Wisniewski PJ II (2017) Exercise is a novel promoter of intestinal health and microbial diversity. Exerc Sport Sci Rev 45:41-47.

Cani PD and de Vos WM (2017) Next-generation beneficial microbes: the case of Akkermansia muciniphila. Front Microbiol 8:1765.

Cannell RJ, Rashid T, Ismail IM, Sidebottom PJ, Knaggs AR, and Marshall PS (1997) Novel metabolites of warfarin produced by Beauveria bassiana and Streptomyces rimosus: a novel application of hplc-nmr. Xenobiotica 27:147-157.

Cano-Garrido O, Seras-Franzoso J, and Garcia-Fruitós E (2015) Lactic acid bacteria: reviewing the potential of a promising delivery live vector for biomedical purposes. Microb Cell Fact 14:137.

Carmody RN and Turnbaugh PJ (2014) Host-microbial interactions in the metabolism of therapeutic and diet-derived xenobiotics. J Clin Invest 124:4173-4181.

Chen JJ, Zeng BH, Li WW, Zhou CJ, Fan SH, Cheng K, Zeng L, Zheng P, Fang L, Wei $\mathrm{H}$, et al. (2017) Effects of gut microbiota on the microRNA and mRNA expression in the hippocampus of mice. Behav Brain Res 322:34-41.

Chen ML, Yi L, Zhang Y, Zhou X, Ran L, Yang J, Zhu JD, Zhang QY, and Mi MT (2016) Resveratrol attenuates trimethylamine-N-oxide (TMAO)-induced atherosclerosis by regulating TMAO synthesis and bile acid metabolism via remodeling of the gut microbiota. MBio 7:e02210-e02215.

Chilloux J and Dumas ME (2017) Are gut microbes responsible for post-dieting weight rebound? Cell Metab 25:6-7.

Chung KT, Stevens SE Jr, and Cerniglia CE (1992) The reduction of azo dyes by the intestinal microflora. Crit Rev Microbiol 18:175-190.

Claesson MJ, Clooney AG, and O'Toole PW (2017) A clinician's guide to microbiome analysis. Nat Rev Gastroenterol Hepatol 14:585-595.

Claesson MJ, Jeffery IB, Conde S, Power SE, O'Connor EM, Cusack S, Harris HM, Coakley M, Lakshminarayanan B, O'Sullivan O, et al. (2012) Gut microbiota composition correlates with diet and health in the elderly. Nature 488:178-184.

Clark RI and Walker DW (2018) Role of gut microbiota in aging-related health decline: insights from invertebrate models. Cell Mol Life Sci 75:93-101.

Clarke G, O'Mahony SM, Dinan TG, and Cryan JF (2014a) Priming for health: gut microbiota acquired in early life regulates physiology, brain and behaviour. Acto Paediatr 103:812-819.

Clarke G, Stilling RM, Kennedy PJ, Stanton C, Cryan JF, and Dinan TG (2014b) Minireview: gut microbiota: the neglected endocrine organ. Mol Endocrinol 28: 1221-1238.

Claus SP, Guillou H, and Ellero-Simatos S (2016) The gut microbiota: a major player in the toxicity of environmental pollutants? NPJ Biofilms Microbiomes 2 16003.

Clayton TA, Baker D, Lindon JC, Everett JR, and Nicholson JK (2009) Pharmacometabonomic identification of a significant host-microbiome metabolic interaction affecting human drug metabolism. Proc Natl Acad Sci USA 106:14728-14733.

Cohen LJ, Esterhazy D, Kim SH, Lemetre C, Aguilar RR, Gordon EA, Pickard AJ, Cross JR, Emiliano AB, Han SM, et al. (2017) Commensal bacteria make GPCR ligands that mimic human signalling molecules. Nature 549:48-53.

Cole CB, Fuller R, Mallet AK, and Rowland IR (1985) The influence of the host on expression of intestinal microbial enzyme activities involved in metabolism of foreign compounds. J Appl Bacteriol 59:549-553.

Cotillard A, Kennedy SP, Kong LC, Prifti E, Pons N, Le Chatelier E, Almeida M, Quinquis B, Levenez F, Galleron N, et al.; ANR MicroObes Consortium (2013) Dietary intervention impact on gut microbial gene richness. Nature 500:585-588.

Craciun S, Marks JA, and Balskus EP (2014) Characterization of choline trimethylamine-lyase expands the chemistry of glycyl radical enzymes. ACS Chem Biol 9:1408-1413. 
Cuhra M, Bøhn T, and Cuhra P (2016) Glyphosate: too much of a good thing? Front Environ Sci 4:1-14.

Das A, Srinivasan M, Ghosh TS, and Mande SS (2016) Xenobiotic metabolism and gut microbiomes. PLoS One 11:e0163099.

Davenport ER, Sanders JG, Song SJ, Amato KR, Clark AG, and Knight R (2017) The human microbiome in evolution. BMC Biol 15:127.

Davey KJ, Cotter PD, O'Sullivan O, Crispie F, Dinan TG, Cryan JF, and O'Mahony SM (2013) Antipsychotics and the gut microbiome: olanzapine-induced metabolic dysfunction is attenuated by antibiotic administration in the rat. Transl Psychiatry 3:e309.

Davey KJ, O’Mahony SM, Schellekens H, O’Sullivan O, Bienenstock J, Cotter PD, Dinan TG, and Cryan JF (2012) Gender-dependent consequences of chronic olanzapine in the rat: effects on body weight, inflammatory, metabolic and microbiota parameters. Psychopharmacology (Berl) 221:155-169.

Davis PJ and Rizzo JD (1982) Microbial transformations of warfarin: stereoselective reduction by Nocardia corallina and Arthrobacter species. Appl Environ Microbiol 43:884-890.

Dawson LF, Donahue EH, Cartman ST, Barton RH, Bundy J, McNerney R, Minton $\mathrm{NP}$, and Wren BW (2011) The analysis of para-cresol production and tolerance in Clostridium difficile 027 and 012 strains. BMC Microbiol 11:86.

de Groot PF, Frissen MN, de Clercq NC, and Nieuwdorp M (2017) Fecal microbiota transplantation in metabolic syndrome: history, present and future. Gut Microbes 8:253-267.

De La Huerga J, Gyorgy P, Waldstein S, Katz R, and Popper H (1953) The effects of antimicrobial agents upon choline degradation in the intestinal tract. $J$ Clin Invest 32:1117-1120.

De La Huerga $J$ and Popper H (1951) Urinary excretion of choline metabolites following choline administration in normals and patients with hepatobiliary diseases. $J$ Clin Invest 30:463-470.

Desbonnet L, Garrett L, Clarke G, Bienenstock J, and Dinan TG (2008) The probiotic Bifidobacteria infantis: an assessment of potential antidepressant properties in the rat. J Psychiatr Res 43:164-174.

Dikongué E and Ségurel L (2017) Latitude as a co-driver of human gut microbial diversity? BioEssays 39:1-6.

Dinan TG and Cryan JF (2017) Gut instincts: microbiota as a key regulator of brain development, ageing and neurodegeneration. J Physiol 595:489-503.

Dinan TG, Stanton C, and Cryan JF (2013) Psychobiotics: a novel class of psychotropic. Biol Psychiatry 74:720-726.

Donaldson GP, Lee SM, and Mazmanian SK (2016) Gut biogeography of the bacterial microbiota. Nat Rev Microbiol 14:20-32.

Donovan MD, Abduljalil K, Cryan JF, Boylan GB, and Griffin BT (2018) Application of a physiologically-based pharmacokinetic model for the prediction of bumetanide plasma and brain concentrations in the neonate. Biopharm Drug Dispos 39: 125-134.

Dumas ME, Rothwell AR, Hoyles L, Aranias T, Chilloux J, Calderari S, Noll EM, Péan N, Boulangé CL, Blancher C, et al. (2017) Microbial-host co-metabolites are prodromal markers predicting phenotypic heterogeneity in behavior, obesity, and impaired glucose tolerance. Cell Reports 20:136-148.

Eade E, Cooper R, and Mitchell AR (2013) Digoxin: time to take the gloves off? Int $J$ Cardiol 164:365-367.

El Aidy S, van den Bogert B, and Kleerebezem M (2015) The small intestine microbiota, nutritional modulation and relevance for health. Curr Opin Biotechnol 32:14-20.

Elmer GW and Remmel RP (1984) Role of the intestinal microflora in clonazepam metabolism in the rat. Xenobiotica 14:829-840.

ElRakaiby M, Dutilh BE, Rizkallah MR, Boleij A, Cole JN, and Aziz RK (2014) Pharmacomicrobiomics: the impact of human microbiome variations on systems pharmacology and personalized therapeutics. OMICS 18:402-414.

Enright EF, Gahan CG, Joyce SA, and Griffin BT (2016) The impact of the gut microbiota on drug metabolism and clinical outcome. Yale J Biol Med 89:375-382.

Falony G, Joossens M, Vieira-Silva S, Wang J, Darzi Y, Faust K, Kurilshikov A, Bonder MJ, Valles-Colomer M, Vandeputte D, et al. (2016) Population-level analysis of gut microbiome variation. Science 352:560-564.

Fijlstra M, Ferdous M, Koning AM, Rings EH, Harmsen HJ, and Tissing WJ (2015) Substantial decreases in the number and diversity of microbiota during chemotherapy-induced gastrointestinal mucositis in a rat model. Support Care Cancer 23:1513-1522.

Flandroy L, Poutahidis T, Berg G, Clarke G, Dao MC, Decaestecker E, Furman E, Haahtela T, Massart S, Plovier H, et al. (2018) The impact of human activities and lifestyles on the interlinked microbiota and health of humans and of ecosystems. Sci Total Environ 627:1018-1038.

Forbes JD, Van Domselaar G, and Bernstein CN (2016) The gut microbiota in immune-mediated inflammatory diseases. Front Microbiol 7:1081.

Forde A and Hill C (2017) Phages of life: the path to pharma. Br J Pharmacol 175: 412-418.

Forslund K, Hildebrand F, Nielsen T, Falony G, Le Chatelier E, Sunagawa S, Prifti E, Vieira-Silva S, Gudmundsdottir V, Pedersen HK, et al.; MetaHIT Consortium (2015) Disentangling type 2 diabetes and metformin treatment signatures in the human gut microbiota. Nature 528:262-266.

Foster JA, Rinaman L, and Cryan JF (2017) Stress \& the gut-brain axis: regulation by the microbiome. Neurobiol Stress 7:124-136.

Fraher MH, O'Toole PW, and Quigley EM (2012) Techniques used to characterize the gut microbiota: a guide for the clinician. Nat Rev Gastroenterol Hepatol 9:312-322.

Friedman ES, Bittinger K, Esipova TV, Hou L, Chau L, Jiang J, Mesaros C, Lund PJ, Liang X, FitzGerald GA, et al. (2018) Microbes vs. chemistry in the origin of the anaerobic gut lumen. Proc Natl Acad Sci USA 115:4170-4175.

Fujii J, Inotsume N, and Nakano M (1987) Degradation of bromazepam by the intestinal microflora. Chem Pharm Bull (Tokyo) 35:4338-4341.

Galley JD, Parry NM, Ahmer BMM, Fox JG, and Bailey MT (2017) The commensal microbiota exacerbate infectious colitis in stressor-exposed mice. Brain Behav Immun 60:44-50.
Galley JD, Yu Z, Kumar P, Dowd SE, Lyte M, and Bailey MT (2014) The structures of the colonic mucosa-associated and luminal microbial communities are distinct and differentially affected by a prolonged murine stressor. Gut Microbes 5:748-760.

Garcia-Gonzalez AP, Ritter AD, Shrestha S, Andersen EC, Yilmaz LS, and Walhout AJM (2017) Bacterial metabolism affects the C. elegans response to cancer chemotherapeutics. Cell 169:431-441.e8.

Gibson GR, Hutkins R, Sanders ME, Prescott SL, Reimer RA, Salminen SJ, Scott K, Stanton C, Swanson KS, Cani PD, et al. (2017) Expert consensus document: the International Scientific Association for Probiotics and Prebiotics (ISAPP) consensus statement on the definition and scope of prebiotics. Nat Rev Gastroenterol Hepatol 14:491-502.

Goldin BR, Peppercorn MA, and Goldman P (1973) Contributions of host and intestinal microflora in the metabolism of L-dopa by the rat. J Pharmacol Exp Ther 186:160-166

Golubeva AV, Crampton S, Desbonnet L, Edge D, O'Sullivan O, Lomasney KW, Zhdanov AV, Crispie F, Moloney RD, Borre YE, et al. (2015) Prenatal stressinduced alterations in major physiological systems correlate with gut microbiota composition in adulthood. Psychoneuroendocrinology 60:58-74

Goodrich JK, Davenport ER, Beaumont M, Jackson MA, Knight R, Ober C, Spector TD, Bell JT, Clark AG, and Ley RE (2016) Genetic determinants of the gut microbiome in UK twins. Cell Host Microbe 19:731-743.

Gopalakrishnan V, Spencer CN, Nezi L, Reuben A, Andrews MC, Karpinets TV, Prieto PA, Vicente D, Hoffman K, Wei SC, et al. (2018) Gut microbiome modulates response to anti-PD-1 immunotherapy in melanoma patients. Science 359:97-103.

Grenham S, Clarke G, Cryan JF, and Dinan TG (2011) Brain-gut-microbe communication in health and disease. Front Physiol 2:94

Guo CJ, Chang FY, Wyche TP, Backus KM, Acker TM, Funabashi M, Taketani M, Donia MS, Nayfach S, Pollard KS, et al. (2017) Discovery of reactive microbiotaderived metabolites that inhibit host proteases. Cell 168:517-526.e18.

Gururajan A, Naughton ME, Scott KA, O'Connor RM, Moloney G, Clarke G, Dowling J, Walsh A, Ismail F, Shorten G, et al. (2016) MicroRNAs as biomarkers for major depression: a role for let-7b and let-7c. Transl Psychiatry 6:e862.

Haft DH and Basu MK (2011) Biological systems discovery in silico: radical S-adenosylmethionine protein families and their target peptides for posttranslational modification. J Bacteriol 193:2745-2755.

Haiser HJ, Gootenberg DB, Chatman K, Sirasani G, Balskus EP, and Turnbaugh PJ (2013) Predicting and manipulating cardiac drug inactivation by the human gut bacterium Eggerthella lenta. Science 341:295-298.

Haiser HJ, Seim KL, Balskus EP, and Turnbaugh PJ (2014) Mechanistic insight into digoxin inactivation by Eggerthella lenta augments our understanding of its pharmacokinetics. Gut Microbes 5:233-238.

Hall SD, Thummel KE, Watkins PB, Lown KS, Benet LZ, Paine MF, Mayo RR, Turgeon DK, Bailey DG, Fontana RJ, et al. (1999) Molecular and physical mechanisms of first-pass extraction. Drug Metab Dispos 27:161-166.

Hata T, Asano Y, Yoshihara K, Kimura-Todani T, Miyata N, Zhang XT, Takakura S, Aiba Y, Koga Y, and Sudo N (2017) Regulation of gut luminal serotonin by commensal microbiota in mice. PLoS One 12:e0180745.

Haynes K, Heitjan D, Kanetsky P, and Hennessy S (2008) Declining public health burden of digoxin toxicity from 1991 to 2004. Clin Pharmacol Ther 84:90-94.

Hehemann JH, Correc G, Barbeyron T, Helbert W, Czjzek M, and Michel G (2010) Transfer of carbohydrate-active enzymes from marine bacteria to Japanese gut microbiota. Nature 464:908-912.

Herles C, Braune A, and Blaut M (2004) First bacterial chalcone isomerase isolated from Eubacterium ramulus. Arch Microbiol 181:428-434.

Hill CJ, Lynch DB, Murphy K, Ulaszewska M, Jeffery IB, O'Shea CA, Watkins C, Dempsey E, Mattivi F, Tuohy K, et al. (2017) Evolution of gut microbiota composition from birth to 24 weeks in the INFANTMET Cohort. Microbiome 5:4.

Hill DR and Spence JR (2016) Gastrointestinal organoids: understanding the molecular basis of the host-microbe interface. Cell Mol Gastroenterol Hepatol 3: 138-149.

Hoban AE, Stilling RM, M Moloney G, Moloney RD, Shanahan F, Dinan TG, Cryan $\mathrm{JF}$, and Clarke G (2017) Microbial regulation of microRNA expression in the amygdala and prefrontal cortex. Microbiome 5:102.

Hoyles L, Fernández-Real JM, Federici M, Serino M, Abbott J, Charpentier J, Heymes C, Luque JL, Anthony E, Barton RH, et al. (2018a) Molecular phenomics and metagenomics of hepatic steatosis in non-diabetic obese women. Nat Med 24: $1070-1080$

Hoyles L, Jiménez-Pranteda ML, Chilloux J, Brial F, Myridakis A, Aranias T, Magnan C, Gibson GR, Sanderson JD, Nicholson JK, et al. (2018b) Metabolic retroconversion of trimethylamine N-oxide and the gut microbiota. Microbiome 6:73.

Hoyles L, Snelling T, Umlai UK, Nicholson JK, Carding SR, Glen RC, and McArthur S (2018c) Microbiome-host systems interactions: protective effects of propionate upon the blood-brain barrier. Microbiome 6:55.

Human Microbiome Project Consortium (2012) Structure, function and diversity of the healthy human microbiome. Nature 486:207-214.

Humblot C, Murkovic M, Rigottier-Gois L, Bensaada M, Bouclet A, Andrieux C, Anba $\mathrm{J}$, and Rabot S (2007) Beta-glucuronidase in human intestinal microbiota is necessary for the colonic genotoxicity of the food-borne carcinogen 2-amino3-methylimidazo[4,5-f]quinoline in rats. Carcinogenesis 28:2419-2425.

Imhann F, Bonder MJ, Vich Vila A, Fu J, Mujagic Z, Vork L, Tigchelaar EF, Jankipersadsing SA, Cenit MC, Harmsen HJ, et al. (2016) Proton pump inhibitors affect the gut microbiome. Gut 65:740-748.

Jacka FN (2017) Nutritional psychiatry: where to next? EBioMedicine 17:24-29.

Jameson E, Doxey AC, Airs R, Purdy KJ, Murrell JC, and Chen Y (2016) Metagenomic data-mining reveals contrasting microbial populations responsible for trimethylamine formation in human gut and marine ecosystems. Microb Genom 2:e000080

Javurek AB, Spollen WG, Johnson SA, Bivens NJ, Bromert KH, Givan SA, and Rosenfeld CS (2016) Effects of exposure to bisphenol A and ethinyl estradiol on the gut microbiota of parents and their offspring in a rodent model. Gut Microbes 7: 471-485. 
Jeffery IB, Lynch DB, and O'Toole PW (2016) Composition and temporal stability of the gut microbiota in older persons. ISME $J$ 10:170-182.

Jezequel SG (1998) Microbial models of mammalian metabolism: uses and misuses (clarification of some misconceptions). J Mol Catal B Enzym 5:371-377.

Jobin C (2018) Precision medicine using microbiota. Science 359:32-34.

Johnson CH, Zhao C, Xu Y, and Mori T (2017) Timing the day: what makes bacterial clocks tick? Nat Rev Microbiol 15:232-242.

Joly C, Gay-Quéheillard J, Léké A, Chardon K, Delanaud S, Bach V, and KhorsiCauet $\mathrm{H}$ (2013) Impact of chronic exposure to low doses of chlorpyrifos on the intestinal microbiota in the Simulator of the Human Intestinal Microbial Ecosystem (SHIME) and in the rat. Environ Sci Pollut Res Int 20:2726-2734.

Jourová L, Anzenbacher P, Lišková B, Matušková Z, Hermanová P, Hudcovic T, Kozáková H, Hrnčírová L, and Anzenbacherová E (2017) Colonization by nonpathogenic bacteria alters mRNA expression of cytochromes $\mathrm{P} 450$ in originally germ-free mice. Folia Microbiol (Praha) 62:463-469.

Joyce SA, Shanahan F, Hill C, and Gahan CG (2014) Bacterial bile salt hydrolase in host metabolism: potential for influencing gastrointestinal microbe-host crosstalk. Gut Microbes 5:669-674.

Kalantar-Zadeh K, Berean KJ, Ha N, Chrimes AF, Xu K, Grando D, Ou JZ, Pillai N, Campbell JL, Brkljača R, et al. (2018) A human pilot trial of ingestible electronic capsules capable of sensing different gases in the gut. Nat Electron 1:79-87.

Kao AC, Spitzer S, Anthony DC, Lennox B, and Burnet PWJ (2018) Prebiotic attenuation of olanzapine-induced weight gain in rats: analysis of central and peripheral biomarkers and gut microbiota. Transl Psychiatry 8:66.

Kassahun K, Mattiuz E, Nyhart E Jr, Obermeyer B, Gillespie T, Murphy A, Goodwin RM, Tupper D, Callaghan JT, and Lemberger L (1997) Disposition and biotransformation of the antipsychotic agent olanzapine in humans. Drug Metab Dispos 25:81-93.

Kelly JR, Allen AP, Temko A, Hutch W, Kennedy PJ, Farid N, Murphy E, Boylan G, Bienenstock J, Cryan JF, et al. (2017) Lost in translation? The potential psychobiotic Lactobacillus rhamnosus (JB-1) fails to modulate stress or cognitive performance in healthy male subjects. Brain Behav Immun 61:50-59.

Kelly JR, Borre Y, O' Brien C, Patterson E, El Aidy S, Deane J, Kennedy PJ, Beers S, Scott K, Moloney G, et al. (2016a) Transferring the blues: depression-associated gut microbiota induces neurobehavioural changes in the rat. $J$ Psychiatr Res 82:109-118.

Kelly JR, Clarke G, Cryan JF, and Dinan TG (2016b) Brain-gut-microbiota axis: challenges for translation in psychiatry. Ann Epidemiol 26:366-372.

Kelly JR, Kennedy PJ, Cryan JF, Dinan TG, Clarke G, and Hyland NP (2015) Breaking down the barriers: the gut microbiome, intestinal permeability and stress-related psychiatric disorders. Front Cell Neurosci 9:392.

Kennedy PJ, Cryan JF, Dinan TG, and Clarke G (2017) Kynurenine pathway metabolism and the microbiota-gut-brain axis. Neuropharmacology 112:399-412.

Kim JK, Choi MS, Jeong JJ, Lim SM, Kim IS, Yoo HH, and Kim DH (2018) Effect of probiotics on pharmacokinetics of orally administered acetaminophen in mice. Drug Metab Dispos 46:122-130.

Klatt NR, Cheu R, Birse K, Zevin AS, Perner M, Noël-Romas L, Grobler A, Westmacott G, Xie IY, Butler J, et al. (2017) Vaginal bacteria modify HIV tenofovir microbicide efficacy in African women. Science 356:938-945.

Kloiber O, Banjac B, and Drewes LR (1988) Protection against acute hyperammonemia: the role of quaternary amines. Toxicology 49:83-90.

Klünemann M, Schmid M, and Patil KR (2014) Computational tools for modeling xenometabolism of the human gut microbiota. Trends Biotechnol 32:157-165.

Koeth RA, Wang Z, Levison BS, Buffa JA, Org E, Sheehy BT, Britt EB, Fu X, Wu Y $\mathrm{Li} \mathrm{L}$, et al. (2013) Intestinal microbiota metabolism of L-carnitine, a nutrient in red meat, promotes atherosclerosis. Nat Med 19:576-585.

Konstandi M (2013) Psychophysiological stress: a significant parameter in drug pharmacokinetics. Expert Opin Drug Metab Toxicol 9:1317-1334.

Koppel N, Maini Rekdal V, and Balskus EP (2017) Chemical transformation of xenobiotics by the human gut microbiota. Science 356:1-13.

Korpela K, Salonen A, Vepsäläinen O, Suomalainen M, Kolmeder C, Varjosalo M, Miettinen S, Kukkonen K, Savilahti E, Kuitunen M, et al. (2018) Probiotic supplementation restores normal microbiota composition and function in antibiotictreated and in caesarean-born infants. Microbiome 6:182.

Krämer SD and Testa B (2008) The biochemistry of drug metabolism--an introduction: Part 6. Inter-individual factors affecting drug metabolism. Chem Biodivers 5:2465-2578.

Krämer SD and Testa B (2009) The biochemistry of drug metabolism--an introduction: Part 7. Intra-individual factors affecting drug metabolism. Chem Biodivers 6:1477-1660.

Kristensen NB, Bryrup T, Allin KH, Nielsen T, Hansen TH, and Pedersen O (2016) Alterations in fecal microbiota composition by probiotic supplementation in healthy adults: a systematic review of randomized controlled trials. Genome Med 8:52.

Kurilshikov A, Wijmenga C, Fu J, and Zhernakova A (2017) Host genetics and gut microbiome: challenges and perspectives. Trends Immunol 38:633-647.

Landfald B, Valeur J, Berstad A, and Raa J (2017) Microbial trimethylamine- $N$-oxide as a disease marker: something fishy? Microb Ecol Health Dis 28:1327309.

Laparra JM and Sanz Y (2010) Interactions of gut microbiota with functional food components and nutraceuticals. Pharmacol Res 61:219-225.

Le Bastard Q, Al-Ghalith GA, Grégoire M, Chapelet G, Javaudin F, Dailly E, Batard E, Knights D, and Montassier E (2018) Systematic review: human gut dysbiosis induced by non-antibiotic prescription medications. Aliment Pharmacol Ther 47:332-345.

Le Chatelier E, Nielsen T, Qin J, Prifti E, Hildebrand F, Falony G, Almeida M, Arumugam M, Batto JM, Kennedy S, et al.; MetaHIT Consortium (2013) Richness of human gut microbiome correlates with metabolic markers. Nature 500:541-546.

Leclercq S, de Timary P, Delzenne NM, and Stärkel P (2017) The link between inflammation, bugs, the intestine and the brain in alcohol dependence. Transl Psychiatry 7:e1048.

Lee H and Ko G (2014) Effect of metformin on metabolic improvement and gut microbiota. Appl Environ Microbiol 80:5935-5943.

Lee JH, Wood TK, and Lee J (2015) Roles of indole as an interspecies and interkingdom signaling molecule. Trends Microbiol 23:707-718.

Lehouritis P, Cummins J, Stanton M, Murphy CT, McCarthy FO, Reid G, Urbaniak C, Byrne WL, and Tangney M (2015) Local bacteria affect the efficacy of chemotherapeutic drugs. Sci Rep 5:14554.
Lehtiö L and Goldman A (2004) The pyruvate formate lyase family: sequences, structures and activation. Protein Eng Des Sel 17:545-552.

Leonardi I, Li X, Semon A, Li D, Doron I, Putzel G, Bar A, Prieto D, Rescigno M, McGovern DPB, et al. (2018) CX3CR1 $1^{+}$mononuclear phagocytes control immunity to intestinal fungi. Science 359:232-236.

Li J, Jia H, Cai X, Zhong H, Feng Q, Sunagawa S, Arumugam M, Kultima JR, Prifti E, Nielsen T, et al.; MetaHIT Consortium (2014) An integrated catalog of reference genes in the human gut microbiome. Nat Biotechnol 32:834-841.

Li Q, Korzan WJ, Ferrero DM, Chang RB, Roy DS, Buchi M, Lemon JK, Kaur AW, Stowers L, Fendt M, et al. (2013) Synchronous evolution of an odor biosynthesis pathway and behavioral response. Curr Biol 23:11-20.

Lin XB, Dieleman LA, Ketabi A, Bibova I, Sawyer MB, Xue H, Field CJ, Baracos VE, and Gänzle MG (2012) Irinotecan (CPT-11) chemotherapy alters intestinal microbiota in tumour bearing rats. PLoS One 7:e39764

Liu S, da Cunha AP, Rezende RM, Cialic R, Wei Z, Bry L, Comstock LE, Gandhi R, and Weiner HL (2016) The host shapes the gut microbiota via fecal microRNA. Cell Host Microbe 19:32-43.

Locquet M, Honvo G, Rabenda V, Van Hees T, Petermans J, Reginster JY, and Bruyère $\mathrm{O}$ (2017) Adverse health events related to self-medication practices among elderly: a systematic review. Drugs Aging 34:359-365.

Long SL, Gahan CGM, and Joyce SA (2017) Interactions between gut bacteria and bile in health and disease. Mol Aspects Med 56:54-65.

$\mathrm{Lu} \mathrm{K}$, Mahbub R, and Fox JG (2015) Xenobiotics: interaction with the intestinal microflora. ILAR J 56:218-227.

Luczynski P, McVey Neufeld KA, Oriach CS, Clarke G, Dinan TG, and Cryan JF (2016) Growing up in a bubble: using germ-free animals to assess the influence of the gut microbiota on brain and behavior. Int $J$ Neuropsychopharmacol 19:1-17.

Lynch SV and Pedersen O (2016) The human intestinal microbiome in health and disease. N Engl J Med 375:2369-2379.

Magnúsdóttir S and Thiele I (2018) Modeling metabolism of the human gut microbiome. Curr Opin Biotechnol 51:90-96.

Maier L, Pruteanu M, Kuhn M, Zeller G, Telzerow A, Anderson EE, Brochado AR, Fernandez KC, Dose H, Mori H, et al. (2018) Extensive impact of non-antibiotic drugs on human gut bacteria. Nature 555:623-628.

Marin IA, Goertz JE, Ren T, Rich SS, Onengut-Gumuscu S, Farber E, Wu M, Overall CC, Kipnis J, and Gaultier A (2017) Microbiota alteration is associated with the development of stress-induced despair behavior. Sci Rep 7:43859.

Martin CR and Mayer EA (2017) Gut-brain axis and behavior. Nestle Nutr Inst Workshop Ser 88:45-53.

Martinez-Guryn K, Hubert N, Frazier K, Urlass S, Musch MW, Ojeda P, Pierre JF, Miyoshi J, Sontag TJ, Cham CM, et al. (2018) Small intestine microbiota regulate host digestive and absorptive adaptive responses to dietary lipids. Cell Host Microbe 23:458-469.e5.

Martinussen J and Hammer K (1994) Cloning and characterization of upp, a gene encoding uracil phosphoribosyltransferase from Lactococcus lactis. J Bacteriol 176: 6457-6463.

Maruo T, Sakamoto M, Ito C, Toda T, and Benno Y (2008) Adlercreutzia equolifaciens gen. nov., sp. nov., an equol-producing bacterium isolated from human faeces, and emended description of the genus Eggerthella. Int J Syst Evol Microbiol 58:1221-1227.

Masters KP and Carr BM (2009) Survey of pharmacists and physicians on drug interactions between combined oral contraceptives and broad-spectrum antibiotics. Pharm Pract (Granada) 7:139-144.

Mathur H, Field D, Rea MC, Cotter PD, Hill C, and Ross RP (2017) Bacteriocinantimicrobial synergy: a medical and food perspective. Front Microbiol 8:1205.

Matthies A, Loh G, Blaut M, and Braune A (2012) Daidzein and genistein are converted to equol and 5-hydroxy-equol by human intestinal Slackia isoflavoniconvertens in gnotobiotic rats. J Nutr 142:40-46.

Maurice CF, Haiser HJ, and Turnbaugh PJ (2013) Xenobiotics shape the physiology and gene expression of the active human gut microbiome. Cell 152:39-50.

Maxmen A (2017) Living therapeutics: scientists genetically modify bacteria to deliver drugs. Nat Med 23:5-7.

Maxson T and Mitchell DA (2016) Targeted treatment for bacterial infections: prospects for pathogen-specific antibiotics coupled with rapid diagnostics. Tetrahedron $\mathbf{7 2}$ $3609-3624$

McEwen BS (2017) Neurobiological and systemic effects of chronic stress. Chronic Stress (Thousand Oaks) 1:1-11.

Meinl W, Sczesny S, Brigelius-Flohé R, Blaut M, and Glatt H (2009) Impact of gut microbiota on intestinal and hepatic levels of phase 2 xenobiotic-metabolizing enzymes in the rat. Drug Metab Dispos 37:1179-1186.

Mellis D and Caporali A (2018) MicroRNA-based therapeutics in cardiovascular disease: screening and delivery to the target. Biochem Soc Trans 46:11-21.

Michalopoulos GK (2007) Liver regeneration. J Cell Physiol 213:286-300.

Miller CA, Corbin KD, da Costa KA, Zhang S, Zhao X, Galanko JA, Blevins T, Bennett BJ, O'Connor A, and Zeisel SH (2014) Effect of egg ingestion on trimethylamine-N-oxide production in humans: a randomized, controlled, doseresponse study. Am J Clin Nutr 100:778-786

Million M, Diallo A, and Raoult D (2017) Gut microbiota and malnutrition. Microb Pathog 106:127-138.

Min JS and Bae SK (2017) Prediction of drug-drug interaction potential using physiologically based pharmacokinetic modeling. Arch Pharm Res 40:1356-1379. Miñana MD, Hermenegildo C, Llsansola M, Montoliu C, Grisolía S, and Felipo V (1996) Carnitine and choline derivatives containing a trimethylamine group prevent ammonia toxicity in mice and glutamate toxicity in primary cultures of neurons. $J$ Pharmacol Exp Ther 279:194-199.

Moloney GM, O'Leary OF, Salvo-Romero E, Desbonnet L, Shanahan F, Dinan TG, Clarke G, and Cryan JF (2017) Microbial regulation of hippocampal miRNA expression: implications for transcription of kynurenine pathway enzymes. Behav Brain Res 334:50-54.

Moloney GM, Viola MF, Hoban AE, Dinan TG, and Cryan JF (2018) Faecal microRNAs: indicators of imbalance at the host-microbe interface? Benef Microbes $\mathbf{9}$ : 175-183. 
Moloney RD, Desbonnet L, Clarke G, Dinan TG, and Cryan JF (2014) The microbiome: stress, health and disease. Mamm Genome 25:49-74.

Morgan AP, Crowley JJ, Nonneman RJ, Quackenbush CR, Miller CN, Ryan AK, Bogue MA, Paredes SH, Yourstone S, Carroll IM, et al. (2014) The antipsychotic olanzapine interacts with the gut microbiome to cause weight gain in mouse. PLoS One 9:e115225.

Morrison DJ and Preston T (2016) Formation of short chain fatty acids by the gut microbiota and their impact on human metabolism. Gut Microbes 7:189-200.

Moya A and Ferrer M (2016) Functional redundancy-induced stability of gut microbiota subjected to disturbance. Trends Microbiol 24:402-413.

Munguia J and Nizet V (2017) Pharmacological targeting of the host-pathogen interaction: alternatives to classical antibiotics to combat drug-resistant superbugs. Trends Pharmacol Sci 38:473-488.

Murota K, Nakamura Y, and Uehara M (2018) Flavonoid metabolism: the interaction of metabolites and gut microbiota. Biosci Biotechnol Biochem 82:600-610.

Murphy CD (2015) Drug metabolism in microorganisms. Biotechnol Lett 37:19-28.

Murphy K, O’Sullivan O, Rea MC, Cotter PD, Ross RP, and Hill C (2011) Genome mining for radical SAM protein determinants reveals multiple sactibiotic-like gene clusters. PLoS One 6:e20852.

Nakayama H, Kinouchi T, Kataoka K, Akimoto S, Matsuda Y, and Ohnishi Y (1997) Intestinal anaerobic bacteria hydrolyse sorivudine, producing the high blood concentration of 5-(E)-(2-bromovinyl)uracil that increases the level and toxicity of 5-fluorouracil. Pharmacogenetics 7:35-43.

Narhi LO and Fulco AJ (1986) Characterization of a catalytically self-sufficient 119,000-dalton cytochrome P-450 monooxygenase induced by barbiturates in $B a$ cillus megaterium. J Biol Chem 261:7160-7169.

Nguyen TL, Vieira-Silva S, Liston A, and Raes J (2015) How informative is the mouse for human gut microbiota research? Dis Model Mech 8:1-16.

Niehues M and Hensel A (2009) In-vitro interaction of L-dopa with bacterial adhesins of Helicobacter pylori: an explanation for clinicial differences in bioavailability? $J$ Pharm Pharmacol 61:1303-1307.

Ning T, Gong X, Xie L, and Ma B (2017) Gut microbiota analysis in rats with methamphetamine-induced conditioned place preference. Front Microbiol 8: 1620

Nishiyama T, Ogura K, Okuda H, Suda K, Kato A, and Watabe T (2000) Mechanism-based inactivation of human dihydropyrimidine dehydrogenase by (E)-5-(2-bromovinyl)uracil in the presence of NADPH. Mol Pharmacol 57:899-905.

O'Connor RM, Gururajan A, Dinan TG, Kenny PJ, and Cryan JF (2016) All roads lead to the miRNome: miRNAs have a central role in the molecular pathophysiology of psychiatric disorders. Trends Pharmacol Sci 37:1029-1044.

O'Dwyer M, Peklar J, McCallion P, McCarron M, and Henman MC (2016) Factors associated with polypharmacy and excessive polypharmacy in older people with intellectual disability differ from the general population: a cross-sectional observational nationwide study. BMJ Open 6:e010505.

O'Mahony SM, Clarke G, Borre YE, Dinan TG, and Cryan JF (2015) Serotonin, tryptophan metabolism and the brain-gut-microbiome axis. Behav Brain Res 277 $32-48$

O'Mahony SM, Marchesi JR, Scully P, Codling C, Ceolho AM, Quigley EM, Cryan JF, and Dinan TG (2009) Early life stress alters behavior, immunity, and microbiota in rats: implications for irritable bowel syndrome and psychiatric illnesses. Biol Psychiatry 65:263-267.

O'Sullivan O, Cronin O, Clarke SF, Murphy EF, Molloy MG, Shanahan F, and Cotter PD (2015) Exercise and the microbiota. Gut Microbes 6:131-136.

Oteiza PI, Fraga CG, Mills DA, and Taft DH (2018) Flavonoids and the gastrointestinal tract: local and systemic effects. Mol Aspects Med 61:41-49.

O'Toole PW, Marchesi JR, and Hill C (2017) Next-generation probiotics: the spectrum from probiotics to live biotherapeutics. Nat Microbiol 2:17057.

Otte C, Gold SM, Penninx BW, Pariante CM, Etkin A, Fava M, Mohr DC, and Schatzberg AF (2016) Major depressive disorder. Nat Rev Dis Primers 2:16065

Ou JZ, Yao CK, Rotbart A, Muir JG, Gibson PR, and Kalantar-zadeh K (2015) Human intestinal gas measurement systems: in vitro fermentation and gas capsules. Trends Biotechnol 33:208-213.

Ozturk N, Ozturk D, Kavakli IH, and Okyar A (2017) Molecular aspects of circadian pharmacology and relevance for cancer chronotherapy. Int J Mol Sci 18:1-34.

Pantelidou M, Tsiakitzis K, Rekka EA, and Kourounakis PN (2017) Biologic stress, oxidative stress, and resistance to drugs: what is hidden behind. Molecules 22 $1-13$

Papalini S, Michels F, Kohn N, Wegman J, van Hemert S, Roelofs K, Arias Vasquez A, and Aarts E (2018) Stress matters: a double-blind, randomized controlled trial on the effects of a multispecies probiotic on neurocognition bioRxiv. DOI: 10.1101/263673.

Park GS, Park MH, Shin W, Zhao C, Sheikh S, Oh SJ, and Kim HJ (2017) Emulating host-microbiome ecosystem of human gastrointestinal tract in vitro. Stem Cell Rev 13:321-334.

Patterson AD and Turnbaugh PJ (2014) Microbial determinants of biochemical individuality and their impact on toxicology and pharmacology. Cell Metab 20: $761-768$

Patterson E, Cryan JF, Fitzgerald GF, Ross RP, Dinan TG, and Stanton C (2014) Gut microbiota, the pharmabiotics they produce and host health. Proc Nutr Soc $\mathbf{7 3}$ 477-489.

Pellock SJ and Redinbo MR (2017) Glucuronides in the gut: sugar-driven symbioses between microbe and host. J Biol Chem 292:8569-8576.

Peppercorn MA and Goldman P (1972a) Caffeic acid metabolism by gnotobiotic rats and their intestinal bacteria. Proc Natl Acad Sci USA 69:1413-1415.

Peppercorn MA and Goldman P (1972b) The role of intestinal bacteria in the metabolism of salicylazosulfapyridine. J Pharmacol Exp Ther 181:555-562.

Perez-Muñoz ME, Arrieta MC, Ramer-Tait AE, and Walter J (2017) A critical assessment of the "sterile womb" and "in utero colonization" hypotheses: implications for research on the pioneer infant microbiome. Microbiome 5:48.

Pieper IA and Bertau M (2010) Predictive tools for the evaluation of microbial effects on drugs during gastrointestinal passage. Expert Opin Drug Metab Toxicol 6: $747-760$
Piwowarski JP, Stanisławska I, Granica S, Stefańska J, and Kiss AK (2017) Phase II conjugates of urolithins isolated from human urine and potential role of $\beta$-glucuronidases in their disposition. Drug Metab Dispos 45:657-665.

Pollet RM, D'Agostino EH, Walton WG, Xu Y, Little MS, Biernat KA, Pellock SJ, Patterson LM, Creekmore BC, Isenberg HN, et al. (2017) An atlas of $\beta$-glucuronidases in the human intestinal microbiome. Structure 25:967-977 e5.

Portune KJ, Benítez-Páez A, Del Pulgar EM, Cerrudo V, and Sanz Y (2017) Gut microbiota, diet, and obesity-related disorders: the good, the bad, and the future challenges. Mol Nutr Food Res 61:1-17.

Prenderville JA, Kennedy PJ, Dinan TG, and Cryan JF (2015) Adding fuel to the fire: the impact of stress on the ageing brain. Trends Neurosci 38:13-25.

Pusceddu MM, El Aidy S, Crispie F, O'Sullivan O, Cotter P, Stanton C, Kelly P, Cryan JF, and Dinan TG (2015) N-3 polyunsaturated fatty acids (PUFAs) reverse the impact of early-life stress on the gut microbiota. PLoS One 10:e0139721.

Pusceddu MM, Kelly P, Stanton C, Cryan JF, and Dinan TG (2016) N-3 polyunsaturated fatty acids through the lifespan: implication for psychopathology. Int $J$ Neuropsychopharmacol 19:1-23.

Qin J, Li R, Raes J, Arumugam M, Burgdorf KS, Manichanh C, Nielsen T, Pons N, Levenez F, Yamada T, et al.; MetaHIT Consortium (2010) A human gut microbial gene catalogue established by metagenomic sequencing. Nature 464:59-65.

Qin N, Yang F, Li A, Prifti E, Chen Y, Shao L, Guo J, Le Chatelier E, Yao J, Wu L, et al. (2014) Alterations of the human gut microbiome in liver cirrhosis. Nature 513:59-64

Quigley EMM (2017) Gut microbiome as a clinical tool in gastrointestinal disease management: are we there yet? Nat Rev Gastroenterol Hepatol 14:315-320.

Rajilić-Stojanović M, Jonkers DM, Salonen A, Hanevik K, Raes J, Jalanka J, de Vos WM, Manichanh C, Golic N, Enck P, et al. (2015) Intestinal microbiota and diet in IBS: causes, consequences, or epiphenomena? Am J Gastroenterol 110:278-287.

Rath S, Heidrich B, Pieper DH, and Vital M (2017) Uncovering the trimethylamineproducing bacteria of the human gut microbiota. Microbiome 5:54.

Roberts AB, Wallace BD, Venkatesh MK, Mani S, and Redinbo MR (2013) Molecular insights into microbial $\beta$-glucuronidase inhibition to abrogate CPT-11 toxicity. Mol Pharmacol 84:208-217.

Roberts MS, Magnusson BM, Burczynski FJ, and Weiss M (2002) Enterohepatic circulation: physiological, pharmacokinetic and clinical implications. Clin Pharmacokinet 41:751-790.

Robertson RC, Seira Oriach C, Murphy K, Moloney GM, Cryan JF, Dinan TG, Paul Ross R, and Stanton C (2017a) Omega-3 polyunsaturated fatty acids critically regulate behaviour and gut microbiota development in adolescence and adulthood. Brain Behav Immun 59:21-37.

Robertson RC, Seira Oriach C, Murphy K, Moloney GM, Cryan JF, Dinan TG, Ross $\mathrm{RP}$, and Stanton C (2017b) Deficiency of essential dietary n-3 PUFA disrupts the caecal microbiome and metabolome in mice. Br J Nutr 118:959-970.

Rothschild D, Weissbrod O, Barkan E, Kurilshikov A, Korem T, Zeevi D, Costea PI, Godneva A, Kalka IN, Bar N, et al. (2018) Environment dominates over host genetics in shaping human gut microbiota. Nature 555:210-215.

Routy B, Le Chatelier E, Derosa L, Duong CPM, Alou MT, Daillère R, Fluckiger A Messaoudene M, Rauber C, Roberti MP, et al. (2018) Gut microbiome influences efficacy of PD-1-based immunotherapy against epithelial tumors. Science 359: 91-97.

Ryan PM, London LE, Bjorndahl TC, Mandal R, Murphy K, Fitzgerald GF, Shanahan F, Ross RP, Wishart DS, Caplice NM, et al. (2017) Microbiome and metabolome modifying effects of several cardiovascular disease interventions in apo- $\mathrm{E}^{-/-}$mice. Microbiome 5:30.

Ryan PM, Ross RP, Fitzgerald GF, Caplice NM, and Stanton C (2015) Functional food addressing heart health: do we have to target the gut microbiota? Curr Opin Clin Nutr Metab Care 18:566-571.

Saad R, Rizkallah MR, and Aziz RK (2012) Gut Pharmacomicrobiomics: the tip of an iceberg of complex interactions between drugs and gut-associated microbes. Gut Pathog 4:16.

Saitta KS, Zhang C, Lee KK, Fujimoto K, Redinbo MR, and Boelsterli UA (2014) Bacterial $\beta$-glucuronidase inhibition protects mice against enteropathy induced by indomethacin, ketoprofen or diclofenac: mode of action and pharmacokinetics. Xenobiotica 44:28-35.

Sandhu KV, Sherwin E, Schellekens H, Stanton C, Dinan TG, and Cryan JF (2017) Feeding the microbiota-gut-brain axis: diet, microbiome, and neuropsychiatry. Transl Res 179:223-244.

Sarkar A, Lehto SM, Harty S, Dinan TG, Cryan JF, and Burnet PWJ (2016) Psychobiotics and the manipulation of bacteria-gut-brain signals. Trends Neurosci $\mathbf{3 9}$ $763-781$

Savignac HM, Kiely B, Dinan TG, and Cryan JF (2014) Bifidobacteria exert strainspecific effects on stress-related behavior and physiology in BALB/c mice. Neurogastroenterol Motil 26:1615-1627.

Savignac HM, Tramullas M, Kiely B, Dinan TG, and Cryan JF (2015) Bifidobacteria modulate cognitive processes in an anxious mouse strain. Behav Brain Res 287: $59-72$

Schmidt TM and Kao JY (2014) A little O2 may go a long way in structuring the GI microbiome. Gastroenterology 147:956-959.

Schwarz E, Maukonen J, Hyytiainen T, Kieseppa T, Oresic M, Sabunciyan S, Mantere O, Saarela M, Yolken R, and Suvisaari J (2018) Analysis of microbiota in first episode psychosis identifies preliminary associations with symptom severity and treatment response. Schizophr Res 192:398-403.

Scott KA, Hoban AE, Clarke G, Moloney GM, Dinan TG, and Cryan JF (2015) Thinking small: towards microRNA-based therapeutics for anxiety disorders. $E x$ pert Opin Investig Drugs 24:529-542.

Scott TA, Quintaneiro LM, Norvaisas P, Lui PP, Wilson MP, Leung KY, HerreraDominguez L, Sudiwala S, Pessia A, Clayton PT, et al. (2017) Host-microbe co-metabolism dictates cancer drug efficacy in C. elegans. Cell 169:442-456.e18.

Selwyn FP, Cheng SL, Bammler TK, Prasad B, Vrana M, Klaassen C, and Cui JY (2015a) Developmental regulation of drug-processing genes in livers of germ-free mice. Toxicol Sci 147:84-103. 
Selwyn FP, Cheng SL, Klaassen CD, and Cui JY (2016) Regulation of hepatic drugmetabolizing enzymes in germ-free mice by conventionalization and probiotics. Drug Metab Dispos 44:262-274.

Selwyn FP, Cui JY, and Klaassen CD (2015b) RNA-seq quantification of hepatic drug processing genes in germ-free mice. Drug Metab Dispos 43:1572-1580.

Sender R, Fuchs S, and Milo R (2016a) Are we really vastly outnumbered? Revisiting the ratio of bacterial to host cells in humans. Cell 164:337-340.

Sender R, Fuchs S, and Milo R (2016b) Revised estimates for the number of human and bacteria cells in the body. PLoS Biol 14:e1002533.

Setchell KD, Brown NM, and Lydeking-Olsen E (2002) The clinical importance of the metabolite equol-a clue to the effectiveness of soy and its isoflavones. $J$ Nutr 132: $3577-3584$.

Shanahan F, van Sinderen D, O'Toole PW, and Stanton C (2017) Feeding the microbiota: transducer of nutrient signals for the host. Gut 66:1709-1717.

Sheehan D and Shanahan F (2017) The gut microbiota in inflammatory bowel disease. Gastroenterol Clin North Am 46:143-154.

Simrén M, Barbara G, Flint HJ, Spiegel BM, Spiller RC, Vanner S, Verdu EF, Whorwell PJ, and Zoetendal EG; Rome Foundation Committee (2013) Intestinal microbiota in functional bowel disorders: a Rome foundation report. Gut 62:159-176.

Singh B, Mal G, and Marotta F (2017) Designer probiotics: paving the way to living therapeutics. Trends Biotechnol 35:679-682.

Slaby O, Laga R, and Sedlacek O (2017) Therapeutic targeting of non-coding RNAs in cancer. Biochem J 474:4219-4251.

Smith EA and Macfarlane GT (1997) Formation of phenolic and indolic compounds by anaerobic bacteria in the human large intestine. Microb Ecol 33:180-188.

Smith RV and Rosazza JP (1974) Microbial models of mammalian metabolism: aromatic hydroxylation. Arch Biochem Biophys 161:551-558.

Sohail MU, Althani A, Anwar H, Rizzi R, and Marei HE (2017) Role of the gastrointestinal tract microbiome in the pathophysiology of diabetes mellitus. J Diabetes Res 2017:9631435.

Song HS, Renslow RS, Fredrickson JK, and Lindemann SR (2015) Integrating ecological and engineering concepts of resilience in microbial communities. Front Microbiol 6:1298.

Sonnenburg ED, Smits SA, Tikhonov M, Higginbottom SK, Wingreen NS, and Sonnenburg JL (2016) Diet-induced extinctions in the gut microbiota compound over generations. Nature 529:212-215.

Sousa T, Paterson R, Moore V, Carlsson A, Abrahamsson B, and Basit AW (2008) The gastrointestinal microbiota as a site for the biotransformation of drugs. Int $J$ Pharm 363:1-25.

Spanogiannopoulos P, Bess EN, Carmody RN, and Turnbaugh PJ (2016) The microbial pharmacists within us: a metagenomic view of xenobiotic metabolism. Nat Rev Microbiol 14:273-287.

Steidler L, Hans W, Schotte L, Neirynck S, Obermeier F, Falk W, Fiers W, and Remaut E (2000) Treatment of murine colitis by Lactococcus lactis secreting interleukin-10. Science 289:1352-1355.

Stevenson DE and Hurst RD (2007) Polyphenolic phytochemicals--just antioxidants or much more? Cell Mol Life Sci 64:2900-2916.

Stremmel W, Schmidt KV, Schuhmann V, Kratzer F, Garbade SF, Langhans CD, Fricker G, and Okun JG (2017) Blood trimethylamine-N-oxide originates from microbiota mediated breakdown of phosphatidylcholine and absorption from small intestine. PLoS One 12:e0170742.

Suez J, Korem T, Zeevi D, Zilberman-Schapira G, Thaiss CA, Maza O, Israeli D, Zmora N, Gilad S, Weinberger A, et al. (2014) Artificial sweeteners induce glucose intolerance by altering the gut microbiota. Nature 514:181-186.

Suez J, Korem T, Zilberman-Schapira G, Segal E, and Elinav E (2015) Non-caloric artificial sweeteners and the microbiome: findings and challenges. Gut Microbes 6 $149-155$.

Sung MM, Kim TT, Denou E, Soltys CM, Hamza SM, Byrne NJ, Masson G, Park H, Wishart DS, Madsen KL, et al. (2017) Improved glucose homeostasis in obese mice treated with resveratrol is associated with alterations in the gut microbiome. Diabetes 66:418-425.

Takeno S and Sakai T (1991) Involvement of the intestinal microflora in nitrazepaminduced teratogenicity in rats and its relationship to nitroreduction. Teratology 44 209-214.

Tamura G, Gold C, Ferro-Luzzi A, and Ames BN (1980) Fecalase: a model for activation of dietary glycosides to mutagens by intestinal flora. Proc Natl Acad Sci USA 77:4961-4965.

Tanca A, Abbondio M, Palomba A, Fraumene C, Manghina V, Cucca F, Fiorillo E, and Uzzau S (2017) Potential and active functions in the gut microbiota of a healthy human cohort. Microbiome 5:79.

Tarazona JV, Court-Marques D, Tiramani M, Reich H, Pfeil R, Istace F, and Crivellente F (2017) Glyphosate toxicity and carcinogenicity: a review of the scientific basis of the European Union assessment and its differences with IARC. Arch Toxicol 91:2723-2743.

Thaiss CA, Itav S, Rothschild D, Meijer M, Levy M, Moresi C, Dohnalová L, Braverman S, Rozin S, Malitsky S, et al. (2016a) Persistent microbiome alterations modulate the rate of post-dieting weight regain. Nature 540:544-555.

Thaiss CA, Levy M, Korem T, Dohnalova L, Shapiro H, Jaitin DA, David E, Winter DR, Gury-BenAri M, Tatirovsky E, et al. (2016b) Microbiota diurnal rhythmicity programs host transcriptome oscillations. Cell 167:1495-1510.e12.

Thaiss CA, Zeevi D, Levy M, Zilberman-Schapira G, Suez J, Tengeler AC, Abramson L, Katz MN, Korem T, Zmora N, et al. (2014) Transkingdom control of microbiota diurnal oscillations promotes metabolic homeostasis. Cell 159: $514-529$

Thiele I, Clancy CM, Heinken A, and Fleming RMT (2017) Quantitative systems pharmacology and the personalized drug-microbiota-diet axis. Curr Opin Syst Biol 4:43-52.

Toda T, Ohi K, Kudo T, Yoshida T, Ikarashi N, Ito K, and Sugiyama K (2009a) Ciprofloxacin suppresses Cyp3a in mouse liver by reducing lithocholic acidproducing intestinal flora. Drug Metab Pharmacokinet 24:201-208.
Toda T, Saito N, Ikarashi N, Ito K, Yamamoto M, Ishige A, Watanabe K, and Sugiyama K (2009b) Intestinal flora induces the expression of Cyp3a in the mouse liver. Xenobiotica 39:323-334.

Torres-Fuentes C, Schellekens H, Dinan TG, and Cryan JF (2017) The microbiotagut-brain axis in obesity. Lancet Gastroenterol Hepatol 2:747-756.

Tralau T and Luch A (2013) The evolution of our understanding of endo-xenobiotic crosstalk and cytochrome P450 regulation and the therapeutic implications. Expert Opin Drug Metab Toxicol 9:1541-1554

Tralau T, Sowada J, and Luch A (2015) Insights on the human microbiome and its xenobiotic metabolism: what is known about its effects on human physiology? Expert Opin Drug Metab Toxicol 11:411-425.

Tropini C, Earle KA, Huang KC, and Sonnenburg JL (2017) The gut microbiome: connecting spatial organization to function. Cell Host Microbe 21:433-442.

Tsuji H, Moriyama K, Nomoto K, Miyanaga N, and Akaza H (2010) Isolation and characterization of the equol-producing bacterium Slackia sp. strain NATTS. Arch Microbiol 192:279-287.

Turnbaugh PJ, Hamady M, Yatsunenko T, Cantarel BL, Duncan A, Ley RE, Sogin ML, Jones WJ, Roe BA, Affourtit JP, et al. (2009) A core gut microbiome in obese and lean twins. Nature 457:480-484.

van Hogezand RA, Kennis HM, van Schaik A, Koopman JP, van Hees PA, and van Tongeren JH (1992) Bacterial acetylation of 5-aminosalicylic acid in faecal suspensions cultured under aerobic and anaerobic conditions. Eur J Clin Pharmacol 43:189-192.

Voigt RM, Forsyth CB, Green SJ, Engen PA, and Keshavarzian A (2016) Circadian rhythm and the gut microbiome. Int Rev Neurobiol 131:193-205.

Wallace BD, Roberts AB, Pollet RM, Ingle JD, Biernat KA, Pellock SJ, Venkatesh MK, Guthrie L, O'Neal SK, Robinson SJ, et al. (2015) Structure and inhibition of microbiome $\beta$-glucuronidases essential to the alleviation of cancer drug toxicity. Chem Biol 22:1238-1249.

Wallace BD, Wang H, Lane KT, Scott JE, Orans J, Koo JS, Venkatesh M, Jobin C, Yeh LA, Mani S, et al. (2010) Alleviating cancer drug toxicity by inhibiting a bacterial enzyme. Science 330:831-835.

Walsh KA, O'Regan NA, Byrne S, Browne J, Meagher DJ, and Timmons S (2016) Patterns of psychotropic prescribing and polypharmacy in older hospitalized patients in Ireland: the influence of dementia on prescribing. Int Psychogeriatr 28 1807-1820

Wang Z, Klipfell E, Bennett BJ, Koeth R, Levison BS, Dugar B, Feldstein AE, Britt EB, Fu X, Chung YM, et al. (2011) Gut flora metabolism of phosphatidylcholine promotes cardiovascular disease. Nature 472:57-63.

Watson H, Mitra S, Croden FC, Taylor M, Wood HM, Perry SL, Spencer JA, Quirke $\mathrm{P}$, Toogood GJ, Lawton CL, et al. (2018) A randomised trial of the effect of omega-3 polyunsaturated fatty acid supplements on the human intestinal microbiota. Gut 67:1974-1983.

Williams SC (2014) Gnotobiotics. Proc Natl Acad Sci USA 111:1661.

Williamson G and Clifford MN (2017) Role of the small intestine, colon and microbiota in determining the metabolic fate of polyphenols. Biochem Pharmacol 139:24-39.

Wilson CJ, Zhan H, Swint-Kruse L, and Matthews KS (2007) The lactose repressor system: paradigms for regulation, allosteric behavior and protein folding. Cell Mol Life Sci 64:3-16.

Wilson ID and Nicholson JK (2017) Gut microbiome interactions with drug metabolism, efficacy, and toxicity. Transl Res 179:204-222.

Winek K, Dirnagl U, and Meisel A (2016) The gut microbiome as therapeutic target in central nervous system diseases: implications for stroke. Neurotherapeutics 13: $762-774$.

Wlodarska M, Luo C, Kolde R, d'Hennezel E, Annand JW, Heim CE, Krastel P, Schmitt EK, Omar AS, Creasey EA, et al. (2017) Indoleacrylic acid produced by commensal peptostreptococcus species suppresses inflammation. Cell Host Microbe 22:25-37.e6.

Wu H, Esteve E, Tremaroli V, Khan MT, Caesar R, Mannerås-Holm L, Ståhlman M, Olsson LM, Serino M, Planas-Fèlix M, et al. (2017) Metformin alters the gut microbiome of individuals with treatment-naive type 2 diabetes, contributing to the therapeutic effects of the drug. Nat Med 23:850-858.

Xu Y, Xie Z, Wang H, Shen Z, Guo Y, Gao Y, Chen X, Wu Q, Li X, and Wang K (2017) Bacterial diversity of intestinal microbiota in patients with substance use disorders revealed by 16S rRNA gene deep sequencing. Sci Rep 7:3628.

Yan A, Culp E, Perry J, Lau JT, MacNeil LT, Surette MG, and Wright GD (2018) Transformation of the anticancer drug doxorubicin in the human gut microbiome. ACS Infect Dis 4:68-76.

Yang B, Chen H, Gu Z, Tian F, Ross RP, Stanton C, Chen YQ, Chen W, and Zhang H (2014) Synthesis of conjugated linoleic acid by the linoleate isomerase complex in food-derived lactobacilli. J Appl Microbiol 117:430-439.

Yano JM, Yu K, Donaldson GP, Shastri GG, Ann P, Ma L, Nagler CR, Ismagilov RF, Mazmanian SK, and Hsiao EY (2015) Indigenous bacteria from the gut microbiota regulate host serotonin biosynthesis. Cell 161:264-276.

Yeo HK, Hyun YJ, Jang SE, Han MJ, Lee YS, and Kim DH (2012) Development of fecal microbial enzyme mix for mutagenicity assay of natural products. J Microbiol Biotechnol 22:838-848.

Yoo DH, Kim IS, Van Le TK, Jung IH, Yoo HH, and Kim DH (2014) Gut microbiotamediated drug interactions between lovastatin and antibiotics. Drug Metab Dispos 42:1508-1513.

Yoo HH, Kim IS, Yoo DH, and Kim DH (2016) Effects of orally administered antibiotics on the bioavailability of amlodipine: gut microbiota-mediated drug interaction. J Hypertens 34:156-162.

Zeisel SH and da Costa KA (2009) Choline: an essential nutrient for public health Nutr Rev 67:615-623.

Zhang K and Ni Y (2014) Tyrosine decarboxylase from Lactobacillus brevis: soluble expression and characterization. Protein Expr Purif 94:33-39.

Zheng X, Zhao A, Xie G, Chi Y, Zhao L, Li H, Wang C, Bao Y, Jia W, Luther M, et al. (2013) Melamine-induced renal toxicity is mediated by the gut microbiota. Sci Transl Med 5:172ra22. 
Zhernakova A, Kurilshikov A, Bonder MJ, Tigchelaar EF, Schirmer M, Vatanen T, Mujagic Z, Vila AV, Falony G, Vieira-Silva S, et al.; LifeLines Cohort Study (2016) Population-based metagenomics analysis reveals markers for gut microbiome composition and diversity. Science $\mathbf{3 5 2}$ $565-569$.

Zhu W, Winter MG, Byndloss MX, Spiga L, Duerkop BA, Hughes ER, Büttner L, de Lima Romão E, Behrendt CL, Lopez CA, et al. (2018) Precision editing of the gut microbiota ameliorates colitis. Nature 553:208-211.
Zhu Y, Jameson E, Crosatti M, Schäfer H, Rajakumar K, Bugg TD, and Chen Y (2014) Carnitine metabolism to trimethylamine by an unusual Riesketype oxygenase from human microbiota. Proc Natl Acad Sci USA 111: 4268-4273.

Zoetendal EG, Raes J, van den Bogert B, Arumugam M, Booijink CC, Troost FJ, Bork P, Wels M, de Vos WM, and Kleerebezem M (2012) The human small intestinal microbiota is driven by rapid uptake and conversion of simple carbohydrates. ISME J 6:1415-1426. 